\title{
Group-based generalized q-rung orthopair average aggregation operators and their applications in multi-criteria decision making
}

\author{
Azmat Hussain ${ }^{1} \cdot$ Muhammad Irfan $\mathrm{Ali}^{2} \cdot$ Tahir Mahmood $^{1} \cdot$ Muhammad Munir $^{1}$
}

Received: 11 February 2020 / Accepted: 6 July 2020 / Published online: 4 August 2020

(c) The Author(s) 2020

\begin{abstract}
The objective of this manuscript is to investigate the concept of generalized q-rung orthopair fuzzy sets (Gq- $\mathcal{R O} \mathcal{F} S s)$ and group generalized q-rung orthopair fuzzy sets $(\mathrm{GGq}-\mathcal{R O} \mathcal{F} \mathrm{Ss})$ by incorporating the concept of generalized parameter and group generalized parameters in q-rung orthopair fuzzy environment. The main advantage of generalized parameter in q-rung orthopair fuzzy environment is to reduce uncertain errors in the original information to ensure the expert's level of trust and improve the accuracy of final decision. On the base of generalized parameter, some aggregation operators are introduced such as generalized q-rung orthopair fuzzy average aggregation operators and group generalized q-rung orthopair fuzzy average aggregation operators and studied their related properties. Furthermore, a multi-criteria decision-making method technique based on proposed approach is presented. Finally, a numerical example is provided to illustrate the feasibility of the proposed methods and deliver the sensitivity analysis and comparative analysis, which show the superiority of developed approached than existing methods.
\end{abstract}

Keywords q-rung orthopair fuzzy sets · Gq- $\mathcal{R O} \mathcal{F} S$ s $\cdot$ GGq- $\mathcal{R O} \mathcal{F S s} \cdot \mathrm{Gq}-\mathcal{R O} \mathcal{F}$ aggregation operators $\cdot \mathrm{GGq}-\mathcal{R O} \mathcal{F}$ aggregation operators $\cdot \mathrm{MCDM}$

\section{Introduction}

In real life, decision making plays a significant role and is commonly used to solve real-world problems. Due to the rapid development of human society in technology and many other fields, decision making is a tedious task for the experts to take an intelligent decision. Decision making is a preplan process of selecting the logical choice among several objects. A good decision can change the course of life style. A

$凶$ Azmat Hussain

azmat.phdma66@iiu.edu.pk

Muhammad Irfan Ali

mirfanali13@yahoo.com

Tahir Mahmood

tahirbakhat@iiu.edu.pk

Muhammad Munir

munir.phdma78@iiu.edu.pk

1 Department of Mathematics and Statistics, Faculty of Basic and Applied Sciences, International Islamic University, Islamabad, Pakistan

2 Islamabad Model College for Boys, G-11/1, Islamabad, Pakistan decision maker judges the limitations, advantages and characteristics of each alternatives, and then he could reach to the final decision. In recent era, it becomes difficult for an individual expert to cope with all the decision making information. In many situations of real world, multi-experts are needed for decision making. In recent scenario, multi-criteria decision-making (MCDM) methods play a prominent role in modern decision-making environment, in which one of the most important methods is aggregation operators. Aggregation operators collectively aggregate the attributes and then rank the alternatives to get the most suitable choice. To cope with this uncertain and complex environment Atanassov [3] originated the pioneer notion of intuitionistic fuzzy set (IFS) which deals with the uncertain and complex situations in a better way than the dominant concept of Zadeh [38] fuzzy sets. IFS is characterized in two parts that is membership grade and non-membership grade and this concept based on the sum of membership grade and nonmembership grade must belong to $[0,1]$. Many scholars extended the concept of IFS in several directions in which one of them is aggregation operators which collectively aggregate the information and it is a significant process of decision making. The weighted operators by Yager [30] and ordered weighted aggregation 
operators initiated by Yager and Kacprzyk [35] are used for the fusion of data. Xu [28] originated the concept of IF weighted average $(\mathrm{I} \mathcal{F} \mathcal{W} A)$ operator, IF ordered weighted average (IF $\mathcal{F O} \mathcal{W A}$ ) operator, IF hybrid average (IF $\mathcal{F H A}$ ) operator. Feng et al. [5] presented that the existing definition of generalized intuitionistic fuzzy soft sets is clarified and reformulated as a combination of an intuitionistic fuzzy soft set over the universe of discourse and an intuitionistic fuzzy set in the parameter set. Feng et al. [6] presented a number of lexicographic orders by means of several measures such as the membership, non-membership, score, accuracy and expectation score functions. Some equivalent characterizations and illustrative examples are provided, from which the relationships among these lexicographic orders are ascertained. Ali et al. [2] proposed a graphical ranking method based on the uncertainty index and entropy. For the detail and comprehensive analysis of different aggregation operators in the domain of IFSs are given in $[7,11,18,29,36,37]$. To cope the shortcomings of existing decision-making problems, Zhang et al. [39] investigated the concept of J-divergence and evidential reasoning theory under IF environment and for details, see $[4,20,23]$. However, scholars point out a quite few situations where the dominant concept of IFS failed to cope the circumstances. To handle this deficiency, Yager [31] initiated the remarkable notion of Pythagorean fuzzy $(\mathrm{P} \mathcal{F}) \operatorname{set}(\mathrm{P} \mathcal{F} S)$, whose influential characteristic consists of square sum of membership grade and non-membership belongs to $[0,1]$. On the basis of P $\mathcal{F S ~ [ 3 1 ] , ~ Y a g e r ~ a n d ~ A b a s s o v ~ [ 3 4 ] ~ p r e s e n t e d ~}$ the notion of Pythagorean membership grade in P $\mathcal{F S}$ and they showed their application in decision making. Since the appearance of $\mathrm{P} \mathcal{F} \mathrm{S}$ many practitioners widely extended this remarkable concept in different directions such as, Yager [32] presented the idea of $\mathrm{P} \mathcal{F}$ weighted average $(\mathrm{P} \mathcal{F} \mathcal{W}$ A) operator and $\mathrm{P} \mathcal{F}$ weighted power average operator to aggregate the information, Peng and Yang [24] presented the detail study of division and subtraction operations in $\mathrm{P} \mathcal{F}$ environment, Peng and Yuan [25] investigated the fundamental properties of point aggregation operators and their applications in MCDM, Garg presented the concepts of various aggregation operators in [8-10], Ma and $\mathrm{Xu}$ [22] initiated the notion of symmetric $\mathrm{P} \mathcal{F}$ weighted averaging and geometric $(\mathrm{SP} \mathcal{F} \mathcal{W}$ $\mathrm{A} / \mathrm{G}$ ) operators, Hussain et al. [16] presented rough $\mathrm{P} \mathcal{F}$ ideals in semigroups, Joshi [17] presented the combine study of generalized parameter and $\mathrm{P} \mathcal{F} W A$ operators to construct the concept of generalized $\mathrm{P} \mathcal{F} \mathcal{W}$ A (GP $\mathcal{F} \mathcal{W} A$ ), generalized $\mathrm{P} \mathcal{F}$ ordered weighted average (GPF $\mathcal{F} \mathcal{W A})$, and generalized $\mathrm{PF}$ hybrid average (GPF $\mathcal{F H A}$ ) operators and Hussain et al. [14] proposed the concept of $\mathrm{P} \mathcal{F}$ soft rough set and their desirable properties with detail.

However, in real-life situations, sometimes it is difficult, to take an intelligent decision becomes tedious for the scholars and to point out a quite few situations where the dominant concept of $\mathrm{P} \mathcal{F}$ Ss failed to cope the circumstances. For exam- ple, if the decision maker/expert assigns membership grade 0.8 and non-membership 0.61 , then $(0.8)^{2}+(0.61)^{2}>1$. Recently, to cope with this shortcoming Yager [33] initiated the extensive idea of q-rung orthopair fuzzy $(\mathrm{q}-\mathcal{R O} \mathcal{F})$ set (q$\mathcal{R O} \mathcal{F S}$ ), whose prominent characteristics consists of sum of qth power of membership grade and qth power of nonmembership belongs to [0,1] for $q \geq 1$. So in this case, $(0.8)^{q}+(0.61)^{q}<1$ for $q \geq 3$. It is also observed that the feature of $\mathrm{q}-\mathcal{R O} \mathrm{FS}$ is more stronger than IFS and $\mathrm{P} \mathcal{F} \mathrm{S}$, so it is clear that $\mathrm{q}-\mathcal{R} \mathrm{O} \mathcal{F}$ S is a useful generalization of both IFS and $\mathrm{P} \mathcal{F} \mathrm{S}$. After the initiation of $\mathrm{q}-\mathcal{R} \mathrm{O} \mathcal{F} \mathrm{S}$, quite few contributions to this concept are found in the literature. Ali [1] initiated two new approaches such as L-fuzzy sets and the notion of orbit in $\mathrm{q}-\mathcal{R} \mathrm{O} \mathcal{F}$ environment. Hussain et al. [12] presented covering-based q- $\mathcal{R O} \mathcal{F}$ rough set hybrid with TOPSIS for multi-attribute decision making. Liu and Wang [21] extended the existing approach of aggregation operators to $\mathrm{q}-\mathcal{R O} \mathcal{F}$ environment to get the $\mathrm{q}-\mathcal{R O} \mathcal{F}$ weighted aver-

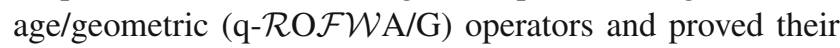
properties. Liu and Liu [19] found the relation between Bonferroni mean operators and $\mathrm{q}-\mathcal{R O} \mathcal{F}$ numbers to achieved different q- $\mathcal{R O} \mathcal{F}$ Bonferroni mean operators. Hussain et al. [15] proposed the concept of $\mathrm{q}-\mathcal{R} \mathrm{O} \mathcal{F}$ soft averaging aggregation operators and presented their desirable properties with detail. The concept of hesitant q- $\mathcal{R O} \mathcal{F}$ aggregation operators was proposed by Hussain et al. [13]. Xing et al. [26] studied the point weighted aggregation operators and Xing et al. [27] presented hamy mean operators in q- $\mathcal{R} O \mathcal{F}$ environment.

It has been observed that all the considered works are accomplished under $\mathrm{q}-\mathcal{R} \mathrm{O} \mathcal{F}$ environments by assuming that the experts are absolutely familiar with the evaluated objects. But in real life, situations like these are partially fulfilled. For example, in MCDM the provided information by experts are completely based on their own choices and may not lead to the accurate decisions. Therefore, acknowledging the described preferences, it is necessary to justify the initial described preferences from other senior expert/judge. In real cases, there are many circumstances where the initial provided preferences need the support of some other senior experts/judge views. For example, in medical diagnose problem consider a person/patient is suffering from an unknown disease and he visits the hospital for the initial treatment to get his disease diagnosed. He describes his preferences in a set of symptoms regarding his physical condition. These symptoms are completely based on information provided by the patient. If the doctor/physician diagnoses the problem according to the patient's preferences without verifying it from another senior expert/doctor's, then he may not be cured well or may be a cause of causality, because the provided information has not been verified from a senior physician/doctor. Therefore, after acknowledging the patient's described symptoms, it is necessary to certify the described information from senior expert physician/doctors. This is only possible by incorporating the 
idea of generalized parameter (in short $\mathcal{G P}$ ) to the original information. The primary provided information by the patient is further verified from another expert physician/senior doctor's and he gives his preference in the form of a generalized parameter. The generalized parameter is itself a $\mathrm{q}-\mathcal{R} O \mathcal{F}$ number $(\mathrm{q}-\mathcal{R} \mathrm{O} \mathcal{F} \mathrm{N})$, which reduces the uncertain information and improves the accuracy of the final decision. Without generalized parameter the initial information described by the patient remains in doubt. Similarly, in MCDM process, to get an intelligent decision, the initially provided preferences from other seniors experts/decision makers need to be verified to reduce the complexity and uncertain errors by incorporating the generalized parameter in the initial information to get the accurate decision.

Hence to cope with such situation, the point of views of other senior expert/observer are needed by incorporating the notion of $\mathcal{G P}$ to the original information. In this paper, we introduced the concept generalized q- $\mathcal{R} O \mathcal{F} \mathrm{S}(\mathrm{Gq}-$ $\mathcal{R O} \mathcal{F S}$ ) by incorporating generalized parameter to views the expertise of other senior decision makers in $\mathrm{q}-\mathcal{R} O \mathcal{F}$ environment, which reduce the complexity and uncertainty errors in original information. Then this idea explored to group generalized parameter where the preferences of two or more senior experts/decision makers are analysed in q$\mathcal{R O} \mathcal{F}$ environments to get new concept of group generalized

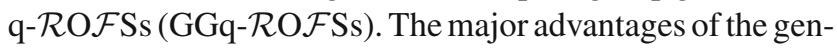
eralized parameter or group generalized parameter is that to reduced probability of complexities, uncertainties and errors in the original information. The main focus of the present work by the application of MCDM, by utilizing generalized parameter and group generalized parameter. For ranking the alternatives, some aggregation operators are introduced for

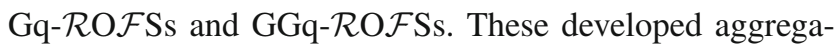
tions operators have the ability to adjust the situations in better sequence on the basis of parameterizations character.

The remaining portions of the manuscript are arranged as. Section 2 consists of a brief review of the existing concepts. Section 3 is devoted for the study of $\mathrm{q}-\mathcal{R O} \mathcal{F}$ ordered

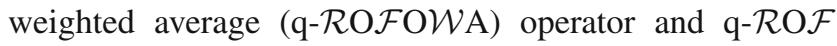

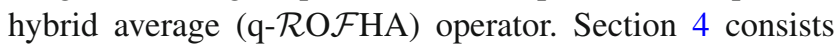
of the definition of generalized $\mathrm{q}-\mathcal{R} \mathrm{O} \mathcal{F} \mathrm{S}$ and the detail study of aggregation operators such as generalized $\mathrm{q}-\mathcal{R} \mathrm{O} \mathcal{F}$ weighted average $(\mathrm{Gq}-\mathcal{R O} \mathcal{F} \mathcal{W} A)$ operator, generalized q$\mathcal{R O} \mathcal{F}$ ordered weighted average $(\mathrm{Gq}-\mathcal{R O} \mathcal{F} \mathrm{OWA})$ operator and generalized $\mathrm{q}-\mathcal{R} \mathrm{O} \mathcal{F}$ hybrid average (Gq- $\mathcal{R O} \mathcal{F} H A)$ operator. In Sect. 5, the defined aggregation operators in Sect. 4 are extended to group generalized $\mathrm{q}-\mathcal{R} O \mathcal{F}$ aggregation operators. Section 6 consists of the MCDM process and a decision algorithm based on the proposed concepts. In Sect. 7, the application of the developed method is presented through an illustrative example. The final Sect. 8 present the comparative remarks of the developed method with existing methods and it has been shown that the developed method is more superior than the existing methods.

\section{Preliminaries}

This section consists of a brief discussion of IFS, PFS and q-ROFSs are given which will help in coming sections.

Definition 1 [3] Let $\mathcal{X}$ be a universal set. An IFS $\mathcal{J}$ on $\mathcal{X}$ can be expressed as

$\mathcal{J}=\left\{<s, \mu_{\mathcal{J}}(s), \eta_{\mathcal{J}}(s)>/ s \in \mathcal{X}\right\}$

where $\mu_{\mathcal{J}}: \mathcal{X} \rightarrow[0,1]$ denotes a membership grade and $\eta_{\mathcal{J}}: \mathcal{X} \rightarrow[0,1]$ denotes a non-membership grade of an object $s \in \mathcal{X}$, to the set $\mathcal{J}$ and it holds that $0 \leq \mu_{\mathcal{J}}(s)+$ $\eta_{\mathcal{J}}(s) \leq 1$.

Definition 2 [31] Consider $\mathcal{X}$ be a universal set. A PFS $\mathcal{J}$ on $\mathcal{X}$ can be expressed as

$\mathcal{J}=\left\{<s, \mu_{\mathcal{J}}(s), \eta_{\mathcal{J}}(s)>/ s \in \mathcal{X}\right\}$,

where $\mu_{\mathcal{J}}: \mathcal{X} \rightarrow[0,1]$ represents a membership grade and $\eta_{\mathcal{J}}: \mathcal{X} \rightarrow[0,1]$ represents a non-membership grade of an object $s \in \mathcal{X}$, to the set $\mathcal{J}$, respectively, and it holds that $0 \leq\left(\mu_{\mathcal{J}}(s)\right)^{2}+\left(\eta_{\mathcal{J}}(s)\right)^{2} \leq 1$. Furthermore, the degree of hesitancy/nondeterminacy is defined as $\pi_{\mathcal{J}}(s)=$ $\sqrt{1-\left(\mu_{\mathcal{J}}(s)\right)^{2}-\left(\eta_{\mathcal{J}}(s)\right)^{2}}$ for all $s \in \mathcal{X}$.

Definition 3 [33] Let $\mathcal{X}$ be a universal set. A q- $\mathcal{R O} \mathcal{F S} \mathcal{J}$ on $\mathcal{X}$ can be expressed as

$\mathcal{J}=\left\{<s, \mu_{\mathcal{J}}(s), \eta_{\mathcal{J}}(s)>_{q} / s \in \mathcal{X}\right\}$

where $\mu_{\mathcal{J}}: \mathcal{X} \rightarrow[0,1]$ denotes a membership grade and $\eta_{\mathcal{J}}: \mathcal{X} \rightarrow[0,1]$ denotes a non-membership grade of an object $s \in \mathcal{X}$, to the set $\mathcal{J}$ respectively and it holds that $0 \leq\left(\mu_{\mathcal{J}}(s)\right)^{q}+\left(\eta_{\mathcal{J}}(s)\right)^{q} \leq 1$ where $q \geq 1$. Further the degree of hesitancy/ nondeterminacy is defined as $\pi_{\mathcal{J}}(s)=$ $\sqrt{1-\left(\mu_{\mathcal{J}}(s)\right)^{q}-\left(\eta_{\mathcal{J}}(s)\right)^{q}}$ for $q \geq 1$ and for all $s \in \mathcal{X}$.

For convenience $\left(\mu_{\mathcal{J}}(s), \eta_{\mathcal{J}}(s)\right)$ is known to be a q$\mathcal{R O} \mathcal{F}$ number $(\mathrm{q}-\mathcal{R O} \mathcal{F} \mathrm{N})$ and is written as $d=\left(\mu_{d}, \eta_{d}\right)$. For any three q- $\mathcal{R O} \mathcal{F}$ Ns $d=\left(\mu_{d}, \eta_{d}\right), d_{1}=\left(\mu_{d_{1}}, \eta_{d_{1}}\right)$ and $d_{2}=\left(\mu_{d_{2}}, \eta_{d_{2}}\right)$, then the basic operation on them are defined as:

i: $d_{1} \cup d_{2}=\left(\max \left(\mu_{d_{1}}, \mu_{d_{2}}\right), \min \left(\eta_{d_{1}}, \eta_{d_{2}}\right)\right)$;

ii: $d_{1} \cap d_{2}=\left(\min \left(\mu_{d_{1}}, \mu_{d_{2}}\right), \max \left(\eta_{d_{1}}, \eta_{d_{2}}\right)\right)$;

iii: $\sim d=\left(\eta_{d}, \mu_{d}\right)$, where $\sim d$ denotes a complement of $d$ 
iv: $d_{1} \oplus d_{2}=\left(\sqrt[q]{\left(\mu_{d_{1}}\right)^{q}+\left(\mu_{d_{2}}\right)^{q}-\mu_{d_{1}}^{q} \mu_{d_{2}}^{q}}, \eta_{d_{1}} \eta_{d_{2}}\right)$;

$\mathrm{v}: d_{1} \otimes d_{2}=\left(\mu_{d_{1}} \mu_{d_{2}}, \sqrt[q]{\left(\eta_{d_{1}}\right)^{q}+\left(\eta_{d_{2}}\right)^{q}-\eta_{d_{1}}^{q} \eta_{d_{2}}^{q}}\right)$;

vi: $\alpha d=\left(\sqrt[q]{1-\left(1-\mu_{d}^{q}\right)^{\alpha}}, \eta_{d}^{\alpha}\right)$;

vii: $d^{\alpha}=\left(\mu_{d}^{\alpha}, \sqrt[q]{1-\left(1-\eta_{d}^{q}\right)^{\alpha}}\right)$.

Definition 4 Suppose $d_{1}=\left(\mu_{d_{1}}, \eta_{d_{1}}\right)$ and $d_{2}=\left(\mu_{d_{2}}, \eta_{d_{2}}\right)$ be two q- $\mathcal{R O} \mathcal{F}$ Ns, then the score function defined by Liu and Wang [21] is given as, $S\left(d_{1}\right)=\mu_{d_{1}}^{q}-\eta_{d_{1}}^{q}$ and $S\left(d_{2}\right)=$ $\mu_{d_{2}}^{q}-\eta_{d_{2}}^{q}$. Similarly the accuracy function of $d_{1}$ and $d_{2}$ are defined as $A\left(d_{1}\right)=\mu_{d_{1}}^{q}+\eta_{d_{1}}^{q}$ and $A\left(d_{2}\right)=\mu_{d_{2}}^{q}+\eta_{d_{2}}^{q}$, respectively.

1: If $S\left(d_{1}\right)<S\left(d_{2}\right)$, then $d_{1}<d_{2}$ that is $d_{1}$ is less than $d_{2}$;

2: If $S\left(d_{1}\right)>S\left(d_{2}\right)$, then $d_{1}>d_{2}$ that is $d_{1}$ is greater than $d_{2}$

if $S\left(d_{1}\right)=S\left(d_{2}\right)$, then larger the accuracy function batter the orthopair is.

On the bases of above operations, Liu and Wang [21] proved the following properties.

Theorem 1 [21] Let $d=\left(\mu_{d}, \eta_{d}\right), d_{1}=\left(\mu_{d_{1}}, \eta_{d_{1}}\right)$ and $d_{2}=\left(\mu_{d_{2}}, \eta_{d_{2}}\right)$ be three $q$-ROFNs and $\alpha, \alpha_{1}, \alpha_{2}>0$, then the following are holds:

i: $d_{1} \oplus d_{2}=d_{2} \oplus d_{1}$;

ii: $d_{1} \otimes d_{2}=d_{2} \otimes d_{1}$;

iii: $\alpha\left(d_{1} \oplus d_{2}\right)=\alpha d_{2} \oplus \alpha d_{1}$;

iv: $\alpha_{1} d \oplus \alpha_{2} d=\left(\alpha_{1}+\alpha_{2}\right) d$;

v: $d^{\alpha_{1}} \otimes d^{\alpha_{2}}=d^{\left(\alpha_{1}+\alpha_{2}\right)}$;

vi: $d_{1}^{\alpha} \otimes d_{2}^{\alpha}=\left(d_{1} \otimes d_{2}\right)^{\alpha}$

\section{q-Rung orthopair fuzzy aggregation operator}

This section is devoted for a brief discussion of aggregation operators such as $\mathrm{q}-\mathcal{R} \mathrm{O} \mathcal{F} \mathcal{W} \mathrm{A}, \mathrm{q}-\mathcal{R O} \mathcal{F} \mathrm{O} \mathcal{W} \mathrm{A}$ and q- $\mathcal{R O} \mathcal{F}$ HA operators.

Definition 5 [21] Consider the collection $d_{\ell}=\left(\mu_{d_{\ell}}, \eta_{d_{\ell}}\right)$ of $n$ q-ROF $\mathcal{F}$ Ns with weight vector $u=\left(u_{1}, u_{2}, \ldots, u_{n}\right)^{\mathrm{T}}$ where $u_{\ell} \in[0,1]$ such that $\sum_{\ell=1}^{n} u_{\ell}=1($ for $\ell=$ $1,2, \ldots, n)$, then the $\mathrm{q}-\mathcal{R} \mathrm{O} \mathcal{F} \mathcal{W}$ A operator is defined as:

$$
\begin{aligned}
& q-\mathcal{R} O \mathcal{F W} A\left(d_{1}, d_{2}, \ldots, d_{n}\right) \\
& =\left(\sqrt[q]{1-\prod_{\ell=1}^{n}\left(1-\mu_{d_{\ell}}^{q}\right)^{u_{\ell}}, \prod_{\ell=1}^{n} \eta_{d_{\ell}}^{u_{\ell}}}\right)
\end{aligned}
$$

Example 1 Suppose four $q$-R $O \mathcal{F} N s d_{1}=(0.8,0.7), d_{2}=$ $(0.9,0.5), d_{3}=(0.7,0.9), d_{4}=(0.6,0.3)$ having weight vector $u=\{0.33,0.15,0.3,0.22\}$ for $q=3$, then

$$
\begin{aligned}
& \sqrt[3]{1-\prod_{\ell=1}^{4}\left(1-\mu_{d_{\ell}}^{3}\right)^{u_{\ell}}}= \\
& \sqrt[3]{1-\left(1-0.8^{3}\right)^{0.33}\left(1-0.9^{3}\right)^{0.15}\left(1-0.7^{3}\right)^{0.3}\left(1-0.6^{3}\right)^{0.22}} \\
& =0.77072 \text { and } \\
& \sqrt[3]{\prod_{\ell=1}^{4} \eta_{d_{\ell}}^{u_{\ell}}}=\left(0.7^{0.33}\right)\left(0.5^{0.15}\right)\left(0.9^{0.3}\right)\left(0.3^{0.22}\right) \\
& =0.595617
\end{aligned}
$$

Now by Definition 5, we have

$$
\begin{aligned}
q- & \mathcal{R} O \mathcal{F W} A\left(d_{1}, d_{2}, d_{3}, d_{4}\right) \\
= & \left(\sqrt[3]{1-\prod_{\ell=1}^{4}\left(1-\mu_{d_{\ell}}^{3}\right)^{u_{\ell}}, \prod_{\ell=1}^{4}\left(\eta_{d_{\ell}}^{u_{\ell}}\right)}\right) \\
= & (0.77072,0.595617)
\end{aligned}
$$

Definition 6 Let us consider the collection $d_{\ell}=\left(\mu_{d_{\ell}}, \eta_{d_{\ell}}\right)$ (for $\ell=1,2, \ldots, n$ ) of $n \mathrm{q}-\mathcal{R O} \mathcal{F} \mathrm{Ns}$, then the q$\mathcal{R O} \mathcal{F O W}$ A operator is given as;

$$
\begin{aligned}
& q-\mathcal{R} O \mathcal{F} O \mathcal{W} A\left(d_{1}, d_{2}, d_{3}, \ldots, d_{n}\right) \\
& \quad=\bigoplus_{\ell=1}^{n} u_{\ell} \tilde{d}_{\ell} \\
& =u_{1} \tilde{d}_{1} \oplus u_{2} \tilde{d}_{2} \oplus \cdots \oplus u_{n} \tilde{d}_{n} .
\end{aligned}
$$

where $\tilde{d}_{\ell}=\left(\mu_{\tilde{d}_{\ell}}, \eta_{\tilde{d}_{\ell}}\right)(\ell=1,2, \ldots, n)$ indicate the $\ell$ th largest object of the collection of $n \mathrm{q}-\mathcal{R O} \mathcal{F}$ Ns $d_{\ell}=$ $\left(\mu_{d_{\ell}}, \eta_{d_{\ell}}\right)$.

The aggregation result of Definition 6 through operation rules is described as in Theorem 2.

Theorem 2 Suppose the collection $d_{\ell}=\left(\mu_{d_{\ell}}, \eta_{d_{\ell}}\right)(\ell=$ $1,2, \ldots, n)$ of $q$-ROF $O \mathcal{F}$ with weight vector $u=$ $\left(u_{1}, u_{2}, \ldots, u_{n}\right)^{\mathrm{T}}$ of $d_{\ell}$ where $u_{\ell} \in[0,1]$ such that $\sum_{\ell=1}^{n} u_{\ell}=1$, then the $q$ - $\mathcal{R} O \mathcal{F} O \mathcal{W} A$ operator is described as:

$$
\begin{aligned}
q & -\mathcal{R O F} O \mathcal{F} A\left(d_{1}, d_{2}, d_{3}, \ldots, d_{n}\right) \\
& =\bigoplus_{\ell=1}^{n} u_{\ell} \tilde{d}_{\ell} \\
& =\left(\sqrt[q]{1-\prod_{\ell=1}^{n}\left(1-\mu_{\tilde{d}_{\ell}}^{q}\right)^{u_{\ell}}}, \prod_{\ell=1}^{n}\left(\eta_{\tilde{d}_{\ell}}^{u_{\ell}}\right)\right),
\end{aligned}
$$

where $\tilde{d}_{\ell}=\left(\mu_{\tilde{d}_{\ell}}, \eta_{\tilde{d}_{\ell}}\right)$ indicate the $\ell$ th largest object of the collection of $n q-\mathcal{R} O \mathcal{F} N s d_{\ell}=\left(\mu_{d_{\ell}}, \eta_{d_{\ell}}\right)(\ell=1,2, \ldots, n)$.

Example 2 Consider four $q$ - $\mathcal{R} O \mathcal{F} N s d_{1}=(0.7,0.3), d_{2}=$ $(0.8,0.65), d_{3}=(0.9,0.6), d_{4}=(0.88,0.7)$ having weight vector $u=(0.3,0.25,0.1,0.35)$ for $q=5$, then to find the score functions of each $\mathrm{q}-\mathcal{R} \mathrm{O} \mathcal{F} \mathrm{Ns}$, that is 
$S\left(d_{1}\right)=0.7^{5}-0.3^{5}=0.166, S\left(d_{2}\right)=0.8^{5}-0.65^{5}=$ $0.212, S\left(d_{3}\right)=0.9^{5}-0.6^{5}=0.513, S\left(d_{4}\right)=0.88^{5}-$ $0.7^{5}=0.360$.

So $S\left(d_{3}\right)>S\left(d_{4}\right)>S\left(d_{2}\right)>S\left(d_{1}\right)$, this implies that $d_{4}>d_{3}>d_{2}>d_{1}$. Thus, $\tilde{d}_{1}=d_{3}, \tilde{d}_{2}=d_{4}, \tilde{d}_{3}=d_{2}$, and $\tilde{d}_{4}=d_{1}$. Further we have

$$
\begin{aligned}
& \sqrt[5]{1-\prod_{\ell=1}^{4}\left(1-\mu_{\tilde{d}_{\ell}}^{5}\right)^{u_{\ell}}}= \\
& \sqrt[5]{1-\left(1-0.9^{5}\right)^{0.3}\left(1-0.88^{5}\right)^{0.25}\left(1-0.8^{5}\right)^{0.1}\left(1-0.7^{5}\right)^{0.35}} \\
&= 0.844094 \\
& \prod_{\ell=1}^{4}\left(\eta_{\tilde{d}_{\ell}}^{u_{\ell}}\right)=\left(0.6^{0.3}\right)\left(0.7^{0.25}\right)\left(0.65^{0.1}\right)\left(0.3^{0.35}\right) \\
&= 0.493178
\end{aligned}
$$

Now by Theorem 2, we have

$$
\begin{aligned}
q & -\mathcal{R} O \mathcal{F} O \mathcal{W} A\left(d_{1}, d_{2}, d_{3}, d_{4}\right) \\
& =\left(\sqrt[5]{1-\prod_{\ell=1}^{4}\left(1-\mu_{\tilde{d}_{\ell}}^{5}\right)^{u_{\ell}}}, \prod_{\ell=1}^{4}\left(\eta_{\tilde{d}_{\ell}}^{u_{\ell}}\right)\right) \\
& =(0.844094,0.493178)
\end{aligned}
$$

Definition 7 Suppose that $d_{\ell}=\left(\mu_{d_{\ell}}, \eta_{d_{\ell}}\right)$ ( for $\ell=$ $1,2, \ldots, n)$, be the collections of q- $\mathcal{R O} \mathcal{F}$ Ns with associated weight vector $u=\left(u_{1}, u_{2}, \ldots, u_{n}\right)^{\mathrm{T}}$ of $d_{\ell}$ where $u_{\ell} \in[0,1]$

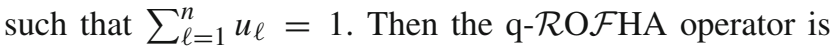
given as:

$$
\begin{aligned}
q- & \mathcal{R} O \mathcal{F} H A\left(d_{1}, d_{2}, d_{3}, \ldots, d_{n}\right) \\
& =\bigoplus_{\ell=1}^{n} u_{\ell} \tilde{d}_{\ell} \\
& =u_{1} \tilde{d}_{1} \oplus u_{2} \tilde{d}_{2} \oplus \cdots \oplus u_{n} \tilde{d}_{n}
\end{aligned}
$$

where $\tilde{d}_{\ell}\left(\tilde{d}_{\ell}=n \hat{u}_{\ell} d_{\ell}\right.$, for $\left.\ell=1,2, \ldots, n\right)$ indicate the $\ell$ th largest object of the collection of $\mathrm{q}-\mathcal{R O} \mathcal{F}$ Ns $d_{\ell}=$ $\left(\mu_{d_{\ell}}, \eta_{d_{\ell}}\right)(\ell=1,2, \ldots, n)$, and $\hat{u}=\left(\hat{u}_{1}, \hat{u}_{2}, \ldots, \hat{u}_{n}\right)^{\mathrm{T}}$ be the weight vector of $d_{\ell}(\ell=1,2, \ldots, n)$ where $\hat{u}_{\ell} \in$ $[0,1]$ such that $\sum_{\ell=1}^{n} \hat{u}_{\ell}=1$ and $n$ indicate the balancing coefficient.

The aggregation result of Definition 7 through operation rules is described as in Theorem 3.

Theorem 3 Suppose $d_{\ell}=\left(\mu_{d_{\ell}}, \eta_{d_{\ell}}\right)(\ell=1,2, \ldots, n)$ be the collection of $q$-R $\mathcal{R} O \mathcal{F} N$, then the $q$ - $\mathcal{R} O \mathcal{F} H A$ operator is described as:

$$
\begin{aligned}
q & -\mathcal{R} O \mathcal{F} H A\left(d_{1}, d_{2}, d_{3}, \ldots, d_{n}\right)=\left(\bigoplus_{\ell=1}^{n} u_{\ell} \tilde{d}_{\ell}\right) \\
& =\left(\sqrt[q]{\left.1-\prod_{\ell=1}^{n}\left(1-\mu_{\tilde{d}_{\ell}}^{q}\right)^{u_{\ell}}, \prod_{\ell=1}^{n}\left(\eta_{\tilde{d}_{\ell}}^{u_{\ell}}\right)\right),}\right.
\end{aligned}
$$

where $\tilde{d}_{\ell}\left(\tilde{d}_{\ell}=n \hat{u}_{\ell} d_{\ell}\right.$, for $\left.\ell=1,2, \ldots, n\right)$ indicate the eth largest object of the collection of $q$-ROFNs $d_{\ell}=$ $\left(\mu_{d_{\ell}}, \eta_{d_{\ell}}\right)(\ell=1,2, \ldots, n)$ and $n$ indicate the balancing coefficient.

Example 3 Suppose four $q$ - $\mathcal{R} O \mathcal{F} N s d_{1}=(0.5,0.2), d_{2}=$ $(0.83,0.6), d_{3}=(0.95,0.65), d_{4}=(0.9,0.75)$ having associated weight vector $u=(0.3,0.2,0.32,0.18)$ for $q=$ 4 , and weight vector $\tilde{u}=(0.4,0.3,0.1,0.2)$, then by using operational law

$n \tilde{u}_{\ell} d_{\ell}=\left(\sqrt[q]{1-\left(1-\mu_{d_{\ell}}^{q}\right)^{n \tilde{u}_{\ell}}}, \eta_{d_{\ell}}^{n \tilde{u}_{\ell}}\right)$

we have

$$
\begin{aligned}
4 \tilde{u}_{1} d_{1} & =\left(\sqrt[4]{1-\left(1-0.5^{4}\right)^{4 \times 0.4}}, 0.2^{4 \times 0.4}\right) \\
& =(0.560,0.076), \\
4 \ddot{u}_{2} d_{2} & =\left(\sqrt[4]{1-\left(1-0.83^{4}\right)^{4 \times 0.3}}, 0.6^{4 \times 0.3}\right) \\
& =(0.856,0.542), \\
4 \tilde{u}_{3} d_{3} & =\left(\sqrt[4]{1-\left(1-0.95^{4}\right)^{4 \times 0.1}}, 0.65^{4 \times 0.1}\right) \\
& =(0.837,0.842), 4 u_{4} d_{4} \\
& =\left(\sqrt[4]{1-\left(1-0.9^{4}\right)^{4 \times 0.2}}, 0.75^{4 \times 0.2}\right) \\
& =(0.870,0.794) .
\end{aligned}
$$

Now to find their score functions, that is

$$
\begin{aligned}
S\left(4 \tilde{u}_{1} d_{1}\right) & =0.560^{4}-0.076^{4}=0.098, \S\left(4 u_{2} d_{2}\right) \\
& =0.856^{4}-0.542^{4} \\
& =0.451, S\left(4 \tilde{u ̈}_{3} d_{3}\right) \\
& =0.837^{4}-0.842^{4} \\
& =-0.012, S\left(4 \tilde{u}_{4} d_{4}\right) \\
& =0.0 .870^{4}-0.794^{4}=-0.396 .
\end{aligned}
$$

So $S\left(4 \ddot{u}_{2} d_{2}\right)>S\left(4 \tilde{u}_{1} d_{1}\right)>S\left(4 \tilde{u}_{3} d_{3}\right)>S\left(4\right.$ ü $\left.4 d_{4}\right)$, this implies that

$$
\begin{gathered}
\tilde{d}_{1}=(0.856,0.542), \tilde{d}_{2}=(0.560,0.076), \\
\tilde{d}_{3}=(0.837,0.842) \text { and } \tilde{d}_{4}=(0.870,0.794)
\end{gathered}
$$

Further, we have

$$
\begin{aligned}
& \sqrt[4]{1-\prod_{\ell=1}^{4}\left(1-\mu_{\tilde{d}_{\ell}}^{4}\right)^{u_{\ell}}}= \\
& \sqrt[4]{\begin{array}{l}
1-\left(1-0.856^{4}\right)^{0.3}\left(1-0.560^{4}\right)^{0.2} \\
\left(1-0.837^{4}\right)^{0.32}\left(1-.870^{4}\right)^{0.18}
\end{array}=0.82465 \text { and }} \\
& \prod_{\ell=1}^{4}\left(\eta_{\tilde{d}_{\ell}}^{u_{\ell}}\right)=\left(0.542^{0.3}\right)\left(0.076^{0.2}\right)\left(0.842^{0.32}\right) \\
& \left(0.794^{0.18}\right)=0.451263
\end{aligned}
$$


Now by Theorem 3 , we have

$$
\begin{aligned}
q & -\mathcal{R} O \mathcal{F} H A\left(d_{1}, d_{2}, d_{3}, d_{4}\right) \\
& =\left(\sqrt[4]{1-\prod_{\ell=1}^{4}\left(1-\mu_{\tilde{d}_{\ell}}^{4}\right)^{u_{\ell}}}, \prod_{\ell=1}^{4}\left(\eta_{\tilde{d}_{\ell}}^{u_{\ell}}\right)\right) \\
& =(0.824648,0.451263)
\end{aligned}
$$

\section{q-Rung orthopair fuzzy averaging aggregation operator under generalized parameter}

In this section, first we will define generalized $\mathrm{q}-\mathcal{R} O \mathcal{F} \mathrm{S}$ and then we will present the detail study of some average aggregation operators under generalized parameter like as $\mathrm{Gq}-\mathcal{R O} \mathcal{F} \mathcal{W}$ A operator, Gq- $\mathcal{R} \mathrm{O} \mathcal{F} O \mathcal{W}$ A operator and Gq$\mathcal{R O} \mathcal{F H A}$ operator and their properties in detail.

\section{q-Rung orthopair fuzzy information under generalized parameter}

Consider a person/patient who is suffering from an unknown disease and he visits the hospital for the initial checkup to diagnose his disease. He describes his preferences in a set of symptoms regarding his physical condition. His preferences are in the form of $\mathrm{q}-\mathcal{R} \mathrm{O} \mathcal{F}$ Ns that is $\mathcal{X}=\left\{d_{1}, d_{2}, d_{3}, d_{4}\right\}$ where $d_{j}(j=1,2,3,4)$ stand for $d_{1}=$ high temperature, $d_{2}=$ headache, $d_{3}=$ cough and $d_{4}=$ constipation, respectively. Let the q- $\mathcal{R O} \mathcal{F} \mathrm{S} \mathcal{X}=\left\{(0.9,0.6)_{\text {hightemperature }}\right.$, $\left.(0.8,0.4)_{\text {headache }}, \quad(0.95,0.5)_{\text {cough }}, \quad(0.7,0.3)_{\text {constipation }}\right\}$ denotes the described symptoms of the patient. These symptoms are completely based on the initial information given by the patient. If the expert means doctor/physician diagnoses the patient according to the patient preferences without verifying it from another senior expert/doctor, then he may not be cured well or may be a cause of causality because the provided information has not been verified from a senior physician/doctor. Therefore, after acknowledging the patient's described symptoms, it is necessary to certify the described information from senior expert physician/doctors. This is only possible by adding the idea of generalized parameter to the original information. The patient information is further verified from another expert physician/senior doctor and he gives their preference with the help of a generalized parameter such as $\hbar=(0.88,0.65)$, then the new $\mathrm{q}-\mathcal{R} \mathrm{O} \mathcal{F}$ S based on generalized parameter is defined as $\mathcal{X}=\{<(0.9,0.6),(0.8,0.4),(0.95,0.5),(0.7,0.3)>$ $(\mathbf{0 . 8 8}, \mathbf{0 . 6 5})\}$. The generalized parameter is a $\mathrm{q}-\mathcal{R} O \mathcal{F} \mathrm{N}$ which deduct the uncertainty and complexity in original information and improves the accuracy of the final decision. Without generalized parameter the initial information described by the patient remains in doubt. Thus, the notion

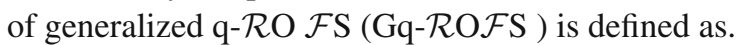

Definition 8 Suppose $\mathcal{X}$ be a universal set. Then a Gq$\mathcal{R O} \mathcal{F S} \mathcal{J}$ of a set $\mathcal{X}$ is defined as

$\mathcal{J}=\left\{<s, \mu_{\mathcal{J}}(s), \eta_{\mathcal{J}}(s)>_{q}\left(\mu_{\hbar}, \eta_{\hbar}\right) / s \in \mathcal{X}\right\}$

where the mappings $\mu_{\mathcal{J}}: \mathcal{X} \rightarrow[0,1]$ and $\eta_{\mathcal{J}}: \mathcal{X} \rightarrow$ $[0,1]$, represents a membership grade and a non-membership grade of $s \in \mathcal{X}$, to the set $\mathcal{J}$, respectively, and satisfying that $0 \leq\left(\mu_{\mathcal{J}}(s)\right)^{q}+\left(\eta_{\mathcal{J}}(s)\right)^{q} \leq 1$ and $\mu_{\hbar}, \eta_{\hbar} \in[0,1]$ represent the degree of truthfulness and falsity grades of q-ROFS $\left\{<s, \mu_{\mathcal{J}}(s), \eta_{\mathcal{J}}(s)>_{q} / s \in \mathcal{X}\right\}$ such that $0 \leq \mu_{\hbar}^{q}+\eta_{\hbar}^{q} \leq 1$ where $q \geq 1$. Further $\hbar=\left(\mu_{\hbar}, \eta_{\hbar}\right)$ is known to be a generalized parameter. This generalized parameter provide the preference assessment of the another senior decision maker/expert. This generalized parameter is itself a q-ROFN.

\section{The generalized q-rung orthopair fuzzy weighted average operator $(\mathrm{Gq}-\mathcal{R} 0 \mathcal{F} \mathcal{W} A)$}

In this subsection, we will present the study of $\mathrm{Gq}-\mathcal{R O} \mathcal{F} \mathcal{W}$ operator and their properties in detail.

Definition 9 Consider a generalized parameter $\hbar=\left(\mu_{\hbar}, \eta_{\hbar}\right)$ for the $\mathrm{q}-\mathcal{R} \mathrm{O} \mathcal{F}$ ss $d_{\ell}=\left(\mu_{d_{\ell}}, \eta_{d_{\ell}}\right)$ (for $\left.\ell=1,2, \ldots, n\right)$, then the Gq- $\mathcal{R O} \mathcal{F} \mathcal{W}$ A operator is defined as;

$$
\begin{gathered}
\mathrm{Gq}-\mathcal{R} O \mathcal{F} \mathcal{W} A\left(<d_{1}, d_{2}, d_{3}, \ldots, d_{n}>, \hbar\right) \\
=\hbar \otimes q-\mathcal{R} O \mathcal{F W} A\left(d_{1}, d_{2}, d_{3}, \ldots, d_{n}\right)
\end{gathered}
$$

The aggregation result for $\mathrm{q}-\mathcal{R} \mathrm{O} \mathcal{F} \mathrm{Ns}$ through operation rules is described as in Theorem 4.

Theorem 4 Suppose the collection $d_{\ell}=\left(\mu_{d_{\ell}}, \eta_{d_{\ell}}\right)(\ell=$ $1,2, \ldots, n)$ of $q$-R $\mathcal{R} O \mathcal{F} N$ s with generalized parameter $\hbar=$ $\left(\mu_{\hbar}, \eta_{\hbar}\right)$ and weight vector $u=\left(u_{1}, u_{2}, \ldots, u_{n}\right)^{\mathrm{T}}$ of $d_{\ell}$ where $u_{\ell} \in[0,1]$ such that $\sum_{\ell=1}^{n} u_{\ell}=1$, then the $G q$ $\mathcal{R} O \mathcal{F}$ WA operator is described as:

$$
\begin{aligned}
& G q-\mathcal{R} O \mathcal{F W} A\left(<d_{1}, d_{2}, d_{3}, \ldots, d_{n}>, \hbar\right) \\
& =\hbar \otimes\left(\bigoplus_{\ell=1}^{n} u_{\ell} d_{\ell}\right) \\
& =\left(\mu_{\hbar} \cdot \sqrt[q]{1-\prod_{\ell=1}^{n}\left(1-\mu_{d_{\ell}}^{q}\right)^{u_{\ell}}}, \sqrt[q]{\eta_{\hbar}^{q}+\left(1-\eta_{\hbar}^{q}\right) \prod_{\ell=1}^{n}\left(\eta_{d_{\ell}}^{u_{\ell}}\right)^{q}}\right)
\end{aligned}
$$

Proof As we know that

$d_{1} \oplus d_{2}=\left(\sqrt[q]{\mu_{d_{1}}^{q}+\mu_{d_{2}}^{q}-\mu_{d_{1}}^{q} \mu_{d_{2}}^{q}}, \eta_{d_{1}} \eta_{d_{2}}\right)$ 
and

$$
\begin{aligned}
u_{1} d_{1} & =\left(\sqrt[q]{1-\left(1-\mu_{d_{1}}^{q}\right)^{u_{1}}}, \eta_{d_{1}}^{u_{1}}\right) \\
\text { and } u_{2} d_{2} & =\left(\sqrt[q]{1-\left(1-\mu_{d_{2}}^{q}\right)^{u_{2}}}, \eta_{d_{2}}^{u_{2}}\right)
\end{aligned}
$$

We use the mathematical induction to prove this theorem. Now for $n=2$ we get

$$
\begin{aligned}
\mathrm{Gq}- & \mathcal{R} O \mathcal{F} \mathcal{W} A\left(<d_{1}, d_{2}>, \hbar\right) \\
& =\hbar \otimes\left(u_{1} d_{1} \oplus u_{2} d_{2}\right) \\
& =\left(\mu_{\hbar}, \eta_{\hbar}\right) \otimes \\
& \left(\sqrt[q]{1-\left(1-\mu_{d_{1}}^{q}\right)^{u_{1}}\left(1-\mu_{d_{2}}^{q}\right)^{u_{2}}}, \eta_{d_{1}}^{u_{1}} \eta_{d_{2}}^{u_{2}}\right) \\
& =\left(\mu_{\hbar}, \eta_{\hbar}\right) \otimes\left(\sqrt[q]{\left.1-\prod_{\ell=1}^{2}\left(1-\mu_{d_{\ell}}^{q}\right)^{u_{\ell}}, \prod_{\ell=1}^{2} \eta_{d_{\ell}}^{u_{\ell}}\right)}\right. \\
& =\left(\begin{array}{c}
\mu_{\hbar} \cdot \sqrt[q]{1-\prod_{\ell=1}^{2}\left(1-\mu_{d_{\ell}}^{q}\right)^{u_{\ell}}}, \\
\left.\sqrt[q]{\eta_{\hbar}^{q}+\left(1-\eta_{\hbar}^{q}\right) \prod_{\ell=1}^{2}\left(\eta_{d_{\ell}}^{u_{\ell}}\right)^{q}}\right)
\end{array}\right.
\end{aligned}
$$

this implies for $n=2$ the result holds. Next suppose that the result true for $n=k$, that is

$$
\begin{aligned}
& \operatorname{Gq}-\mathcal{R} O \mathcal{F} \mathcal{W} A\left(<d_{1}, d_{2}, \ldots, d_{k}>, \hbar\right) \\
& =\hbar \otimes\left(\underset{\ell=1}{\bigoplus} u_{\ell} d_{\ell}\right) \\
& =\left(\mu_{\hbar}, \eta_{\hbar}\right) \otimes\left(\sqrt[q]{1-\prod_{\ell=1}^{k}\left(1-\mu_{d_{\ell}}^{q}\right)^{u_{\ell}}}, \prod_{\ell=1}^{k} \eta_{d_{\ell}}^{u_{\ell}}\right) \\
& =\left(\mu_{\hbar} \cdot \sqrt[q]{1-\prod_{\ell=1}^{k}\left(1-\mu_{d_{\ell}}^{q}\right)^{u_{\ell}}}, \sqrt[q]{\eta_{\hbar}^{q}+\left(1-\eta_{\hbar}^{q}\right) \prod_{\ell=1}^{k}\left(\eta_{d_{\ell}}^{u_{\ell}}\right)^{q}}\right)
\end{aligned}
$$

Now to show that the result hold for $n=k+1$, then we have

$$
\begin{aligned}
& \operatorname{Gq}-\mathcal{R} O \mathcal{F} \mathcal{W} A\left(<d_{1}, d_{2}, \ldots d_{k}, d_{k+1}>, \hbar\right) \\
& =\hbar \otimes\left(\begin{array}{c}
u_{1} d_{1} \oplus u_{2} d_{2} \oplus \cdots \oplus u_{k} d_{k} \oplus \\
u_{k+1} d_{k+1}
\end{array}\right) \\
& =\hbar \otimes\left\{\left(u_{1} d_{1} \oplus u_{2} d_{2} \oplus \cdots \oplus u_{k} d_{k}\right) \oplus u_{k+1} d_{k+1}\right\} \\
& \left.=\left(\mu_{\hbar}, \eta_{\hbar}\right) \otimes\left\{\begin{array}{c}
\left(\sqrt[q]{1-\prod_{\ell=1}^{k}\left(1-\mu_{d_{\ell}}^{q}\right)^{u_{\ell}}}, \prod_{\ell=1}^{k}\left(\eta_{d_{\ell}}^{u_{\ell}}\right)\right.
\end{array}\right) \oplus\right\} \\
& =\left(\mu_{\hbar}, \eta_{\hbar}\right) \otimes\left(\begin{array}{c}
\sqrt[q]{1-\prod_{\ell=1}^{k}\left(1-\mu_{d_{\ell}}^{q}\right)^{u_{\ell}} \cdot\left(1-\mu_{d_{k+1}}^{q}\right)^{u_{k+1}}}, \\
\prod_{\ell=1}^{k}\left(\eta_{d_{\ell}}^{u_{\ell}}\right) \cdot \eta_{d_{k+1}}^{u_{k+1}}
\end{array}\right)
\end{aligned}
$$

$$
\begin{aligned}
& =\left(\mu_{\hbar}, \eta_{\hbar}\right) \otimes\left(\sqrt[q]{\left.1-\prod_{\ell=1}^{k+1}\left(1-\mu_{d_{\ell}}^{q}\right)^{u_{\ell}}, \prod_{\ell=1}^{k+1}\left(\eta_{d_{\ell}}^{u_{\ell}}\right)\right)}\right. \\
& =\left(\mu_{\hbar} \cdot \sqrt[q]{1-\prod_{\ell=1}^{k+1}\left(1-\mu_{d_{\ell}}^{q}\right)^{u_{\ell}}}, \sqrt[q]{\eta_{\hbar}^{q}+\left(1-\eta_{\hbar}^{q}\right) \prod_{\ell=1}^{k+1}\left(\eta_{d_{\ell}}^{u_{\ell}}\right)^{q}}\right)
\end{aligned}
$$

this implies that $n$ is true for $k+1$. Hence, under the study of generalized parameter, the given result hold for any number of q-ROFNs.

Moreover, in the following, it is shown that the aggregated result achieved from $\mathrm{Gq}-\mathcal{R} O \mathcal{F} W A$ is also a $\mathrm{q}-\mathcal{R} O \mathcal{F}$ Ns. Now for any $\ell=1,2,3, \ldots, n$ we have $0 \leq \mu_{d_{\ell}}, \eta_{d_{\ell}} \leq 1$ with $0 \leq \mu_{d_{\ell}}^{q}+\eta_{d_{\ell}}^{q} \leq 1$ for $q \geq 1$. This also implies that

$$
\begin{aligned}
0 & \leq 1-\mu_{d_{\ell}}^{q} \leq 1 \Rightarrow 0 \leq \prod_{\ell=1}^{n}\left(1-\mu_{d_{\ell}}^{q}\right) \leq 1 \Rightarrow 0 \\
& \leq \prod_{\ell=1}^{n}\left(1-\mu_{d_{\ell}}^{q}\right)^{u_{\ell}} \leq 1 \\
& \Rightarrow 0 \leq 1-\prod_{\ell=1}^{n}\left(1-\mu_{d_{\ell}}^{q}\right)^{u_{\ell}} \leq 1 \Rightarrow 0 \\
& \leq \sqrt[q]{1-\prod_{\ell=1}^{n}\left(1-\mu_{d_{\ell}}^{q}\right)^{u_{\ell}}} \leq 1
\end{aligned}
$$

As $\hbar=\left(\mu_{\hbar}, \eta_{\hbar}\right)$ is a generalized parameter where $\mu_{\hbar}, \eta_{\hbar} \in$ $[0,1]$ with $0 \leq \mu_{\hbar}^{q}+\eta_{\hbar}^{q} \leq 1$. Therefore,

$$
\begin{aligned}
& 0 \leq \mu_{\hbar} \cdot \sqrt[q]{1-\prod_{\ell=1}^{n}\left(1-\mu_{d_{\ell}}^{q}\right)^{u_{\ell}}} \leq 1 \text {. Similarly we can show that } \\
& 0 \leq \sqrt[q]{\eta_{\hbar}^{q}+\left(1-\eta_{\hbar}^{q}\right) \prod_{\ell=1}^{n}\left(\eta_{d_{\ell}}^{u_{\ell}}\right)^{q}} \leq 1
\end{aligned}
$$

Furthermore,

$$
\begin{aligned}
0 \leq & \left(\mu_{\hbar} \cdot \sqrt[q]{1-\prod_{\ell=1}^{n}\left(1-\mu_{d_{\ell}}^{q}\right)^{u_{\ell}}}\right)^{q} \\
& +\left(\sqrt[q]{\eta_{\hbar}^{q}+\left(1-\eta_{\hbar}^{q}\right) \prod_{\ell=1}^{n}\left(\eta_{d_{\ell}}^{u_{\ell}}\right)^{q}}\right)^{q} \\
= & \mu_{\hbar}^{q} \cdot\left(1-\prod_{\ell=1}^{n}\left(1-\mu_{d_{\ell}}^{q}\right)^{u_{\ell}}\right.
\end{aligned}
$$




$$
\begin{aligned}
= & \left.\mu_{\hbar}^{q}-\mu_{\hbar}^{q} \prod_{\ell=1}^{n}\left(1-\mu_{d_{\ell}}^{q}\right)^{u_{\ell}}\right)+\eta_{\hbar}^{q} \\
& +\prod_{\ell=1}^{n}\left(\eta_{d_{\ell}}^{u_{\ell}}\right)^{q}-\eta_{\hbar}^{q} \prod_{\ell=1}^{n}\left(\eta_{d_{\ell}}^{u_{\ell}}\right)^{q} \\
= & \left(\mu_{\hbar}^{q}+\eta_{\hbar}^{q}\right)+\prod_{\ell=1}^{n}\left(\eta_{d_{\ell}}^{u_{\ell}}\right)^{q}-\mu_{\hbar}^{q} \prod_{\ell=1}^{n}\left(1-\mu_{d_{\ell}}^{q}\right)^{u_{\ell}} \\
& -\eta_{\hbar}^{q} \prod_{\ell=1}^{n}\left(\eta_{d_{\ell}}^{u_{\ell}}\right)^{q}
\end{aligned}
$$

As $\mu_{d_{\ell}}^{q}+\eta_{d_{\ell}}^{q} \leq 1 \Rightarrow \eta_{d_{\ell}}^{q} \leq 1-\mu_{d_{\ell}}^{q} \Rightarrow-\eta_{d_{\ell}}^{q} \geq-\left(1-\mu_{d_{\ell}}^{q}\right)$

$$
\begin{gathered}
\leq\left(\mu_{\hbar}^{q}+\eta_{\hbar}^{q}\right)+\prod_{\ell=1}^{n}\left(\eta_{d_{\ell}}^{u_{\ell}}\right)^{q}-\mu_{\hbar}^{q} \prod_{\ell=1}^{n}\left(\eta_{d_{\ell}}^{u_{\ell}}\right)^{q} \\
-\eta_{\hbar}^{q} \prod_{\ell=1}^{n}\left(\eta_{d_{\ell}}^{u_{\ell}}\right)^{q}
\end{gathered}
$$$$
=\left(\mu_{\hbar}^{q}+\eta_{\hbar}^{q}\right)+\prod_{\ell=1}^{n}\left(\eta_{d_{\ell}}^{u_{\ell}}\right)^{q}-\left(\mu_{\hbar}^{q}\right.
$$$$
\left.+\eta_{\hbar}^{q}\right) \prod_{\ell=1}^{n}\left(\eta_{d_{\ell}}^{u_{\ell}}\right)^{q}
$$$$
=\left(\mu_{\hbar}^{q}+\eta_{\hbar}^{q}\right)\left(1-\prod_{\ell=1}^{n}\left(\eta_{d_{\ell}}^{u_{\ell}}\right)^{q}\right)+\prod_{\ell=1}^{n}\left(\eta_{d_{\ell}}^{u_{\ell}}\right)^{q}
$$$$
\leq 1-\prod_{\ell=1}^{n}\left(\eta_{d_{\ell}}^{u_{\ell}}\right)^{q}+\prod_{\ell=1}^{n}\left(\eta_{d_{\ell}}^{u_{\ell}}\right)^{q}=1
$$

implies $0 \leq\left(\mu_{\hbar} \cdot \sqrt[q]{1-\prod_{\ell=1}^{n}\left(1-\mu_{d_{\ell}}^{q}\right)^{u_{\ell}}}\right)^{q}$

$$
+\left(\sqrt[q]{\eta_{\hbar}^{q}+\left(1-\eta_{\hbar}^{q}\right) \prod_{\ell=1}^{n}\left(\eta_{d_{\ell}}^{u_{\ell}}\right)^{q}}\right)^{q} \leq 1
$$

Therefore, it is proved that the aggregated result obtained by $G q-\mathcal{R} O \mathcal{F W} A$ operator is also a $q-\mathcal{R} O \mathcal{F} N$.

Remark 1 (a) If the generalized parameter $\hbar=(1,0)$, and $q=1$, then the Gq- $\mathcal{R O} \mathcal{F} \mathcal{W}$ A operator reduces to I $\mathcal{F} \mathcal{W A}$ operator.

(b) If the generalized parameter $\hbar=(1,0)$, and $q=2$, then the $\mathrm{Gq}-\mathcal{R} \mathrm{O} \mathcal{F} \mathcal{W}$ A operator reduces to $\mathrm{P} \mathcal{F} \mathcal{W}$ A operator.

(c) If the value of $q=2$ is fixed then the Gq- $\mathcal{R O} \mathcal{F} \mathcal{W A}$ operator reduces to $\mathrm{GP} \mathcal{F} \mathcal{W}$ A operator.

Example 4 Suppose the generalized parameter $\hbar=$ $(0.9,0.6)$ of four $q$-ROF $N s d_{1}=(0.8,0.7), d_{2}=$ $(0.9,0.5), d_{3}=(0.7,0.9), d_{4}=(0.6,0.3)$ having weight vector $u=\{0.33,0.15,0.3,0.22\}$ for $q=3$, then

$$
\begin{aligned}
& \mu_{\hbar} \cdot \sqrt[3]{1-\prod_{\ell=1}^{4}\left(1-\mu_{d_{\ell}}^{3}\right)^{u_{\ell}}}= \\
& 0.9 \times \sqrt[3]{1-\left(1-0.8^{3}\right)^{0.33}\left(1-0.9^{3}\right)^{0.15}\left(1-0.7^{3}\right)^{0.3}}=0.9 \times \\
& 0.77072=0.69365 \text { and } \\
& \quad \sqrt[3]{\eta_{\hbar}^{3}+\left(1-0.6^{3}\right)^{0.22}}= \\
& \sqrt[3]{0.6^{3}+\left(1-0.6^{3}\right)\left(0.7^{0.33}\right)^{3}\left(0.5^{0.15}\right)^{3}\left(0.9^{0.3}\right)^{3}\left(0.3^{0.22}\right)^{3}}= \\
& 0.72537
\end{aligned}
$$

Now by Theorem 4, we have

$$
\begin{aligned}
\operatorname{Gq} & -\mathcal{R} O \mathcal{F} \mathcal{W} A\left(<d_{1}, d_{2}, d_{3}, d_{4}>, \hbar\right) \\
& =\left(\mu_{\hbar} \cdot \sqrt[3]{1-\prod_{\ell=1}^{4}\left(1-\mu_{d_{\ell}}^{3}\right)^{u_{\ell}}}\right. \\
& \left.\sqrt[3]{\eta_{\hbar}^{3}+\left(1-\eta_{\hbar}^{3}\right) \prod_{\ell=1}^{4}\left(\eta_{d_{\ell}}^{u_{\ell}}\right)^{3}}\right) \\
& =(0.69365,0.72537)
\end{aligned}
$$

Theorem 5 Suppose the collection $d_{\ell}=\left(\mu_{d_{\ell}}, \eta_{d_{\ell}}\right)(\ell=$ $1,2, \ldots, n)$ of $q$ - $\mathcal{R} O \mathcal{F} N s$ with generalized parameter $\hbar=$ $\left(\mu_{\hbar}, \eta_{\hbar}\right)$ and weight vector $u=\left(u_{1}, u_{2}, \ldots, u_{n}\right)^{\mathrm{T}}$ of $d_{\ell}$ where $u_{\ell} \in[0,1]$ such that $\sum_{\ell=1}^{n} u_{\ell}=1$, then for $G q$ $\mathcal{R} O \mathcal{F} \mathcal{W}$ A operator the following are holes:

i: (Idempotency): If $d_{\ell}=d$ (for all $\left.\ell=1,2,3, \ldots, n\right)$, then

$G q-\mathcal{R} O \mathcal{F} \mathcal{W} A\left(<d_{1}, d_{2}, d_{3}, \ldots, d_{n}>, \hbar\right)=\hbar \otimes d$

ii: (Boundary condition): If $d_{\ell}^{-}=\left(\min \mu_{\hbar \otimes d_{\ell}}, \max \eta_{\hbar \otimes d_{\ell}}\right)$ and $d_{\ell}^{+}=\left(\max \mu_{\hbar \otimes d_{\ell}}, \min \eta_{\hbar \otimes d_{\ell}}\right)$ (for all $\ell=$ $1,2,3, \ldots, n)$, then

$d_{\ell}^{-} \leq G q-\mathcal{R} O \mathcal{F W} A\left(<d_{1}, d_{2}, d_{3}, \ldots, d_{n}>, \hbar\right) \leq d_{\ell}^{+}$.

iii: (Monotonicity): Suppose $d_{\ell}=\left(\mu_{d_{\ell}}, \eta_{d_{\ell}}\right)$ and $d_{\ell}^{*}=$ $\left(\mu_{d_{\ell}^{*}}, \eta_{d_{\ell}^{*}}\right)(\ell=1,2, \ldots, n)$ be the collection of $n q-$ ROFNs such that $\mu_{d_{\ell}} \leq \mu_{d_{\ell}^{*}}$ and $\eta_{d_{\ell}} \geq \eta_{d_{\ell}^{*}}$, then

$$
\begin{array}{r}
G q-\mathcal{R} O \mathcal{F W} A\left(<d_{1}, d_{2}, d_{3}, \ldots, d_{n}>, \hbar\right) \leq \\
G q-\mathcal{R O F W} A\left(<d_{1}^{*}, d_{2}^{*}, d_{3}^{*}, \ldots, d_{n}^{*}>, \hbar\right) .
\end{array}
$$

iv: (Commutativity): Suppose $d_{\ell}=\left(\mu_{d_{\ell}}, \eta_{d_{\ell}}\right)$ and $\tilde{d}_{\ell}=$ $\left(\mu_{\tilde{d}_{\ell}}, \eta_{\tilde{d}_{\ell}}\right)(\ell=1,2, \ldots, n)$ be the collection of $n q-$ ROFNs where $\tilde{d}_{\ell}(\ell=1,2, \ldots, n)$ is the lth largest object of $d_{\ell}(\ell=1,2, \ldots, n)$, then

$$
\begin{aligned}
& G q-\mathcal{R} O \mathcal{F W} A\left(<d_{1}, d_{2}, d_{3}, \ldots, d_{n}>, \hbar\right) \\
& =G q-\mathcal{R} O \mathcal{F W} A\left(<\tilde{d}_{1}, \tilde{d}_{2}, \tilde{d}_{3}, \ldots, \tilde{d}_{n}>, \hbar\right) \text {. }
\end{aligned}
$$


Proof i: If $d_{\ell}=d$ (for all $\ell=1,2,3, \ldots, n$ ), then by Theorem 4, we have

$$
\begin{array}{rl}
\mathrm{Gq} & \mathcal{R} O \mathcal{F} \mathcal{W} A\left(<d_{1}, d_{2}, d_{3}, \ldots, d_{n}>, \hbar\right) \\
= & \left(\mu_{\hbar} \cdot \sqrt[q]{1-\prod_{\ell=1}^{n}\left(1-\mu_{d_{\ell}}^{q}\right)^{u_{\ell}}}, \sqrt[q]{\eta_{\hbar}^{q}+\left(1-\eta_{\hbar}^{q}\right) \prod_{\ell=1}^{n}\left(\eta_{d_{\ell}}^{u_{\ell}}\right)^{q}}\right) \\
= & \left(\mu_{\hbar} \cdot \sqrt[q]{1-\prod_{\ell=1}^{n}\left(1-\mu_{d}^{q}\right)^{u_{\ell}}}, \sqrt[q]{\eta_{\hbar}^{q}+\left(1-\eta_{\hbar}^{q}\right) \prod_{\ell=1}^{n}\left(\eta_{d}^{u_{\ell}}\right)^{q}}\right) \\
= & \left(\mu_{\hbar} \cdot \sqrt[q]{1-\left(1-\mu_{d}^{q}\right)^{\sum_{\ell=1}^{n} u_{\ell}}}, \sqrt[q]{\eta_{\hbar}^{q}+\left(1-\eta_{\hbar}^{q}\right)\left(\eta_{d}^{\ell=1} u_{\ell}^{n}\right)^{q}}\right) \\
= & \left(\mu_{\hbar} \cdot \sqrt[q]{\left.1-\left(1-\mu_{d}^{q}\right), \sqrt[q]{\eta_{\hbar}^{q}+\left(1-\eta_{\hbar}^{q}\right)\left(\eta_{d}\right)^{q}}\right)}\right. \\
= & \left(\mu_{\hbar} \cdot \mu_{d}, \sqrt[q]{\eta_{\hbar}^{q}+\left(1-\eta_{\hbar}^{q}\right)\left(\eta_{d}\right)^{q}}\right) \\
= & \hbar d
\end{array}
$$

ii: Let $d_{\ell}^{-}=\left(\min \mu_{\hbar \otimes d_{\ell}}, \max \eta_{\hbar \otimes d_{\ell}}\right)$ and $d_{\ell}^{+}=\left(\max \mu_{\hbar \otimes d_{\ell}}\right.$, $\min \eta_{\hbar \otimes d_{\ell}}$ ) where $\min \mu_{\hbar \otimes d_{\ell}}=\mu_{\hbar}\left(\min \mu_{d_{\ell}}\right), \max \mu_{\hbar \otimes d_{\ell}}=$ $\mu_{\hbar}\left(\max \mu_{d_{\ell}}\right), \max \eta_{\hbar \otimes d_{\ell}}=\left(\eta_{\hbar}^{q}+\left(1-\eta_{\hbar}^{q}\right)\left(\max \eta_{d_{\ell}}\right)^{q}\right)^{\frac{1}{q}}$ and $\min \eta_{\hbar \otimes d_{\ell}}=\left(\eta_{\hbar}^{q}+\left(1-\eta_{\hbar}^{q}\right)\left(\min \eta_{d_{\ell}}\right)^{q}\right)^{\frac{1}{q}}$.

Now for each $\ell=1,2,3, \ldots, n$, we have $\min \mu_{d_{\ell}} \leq$ $\mu_{d_{\ell}} \leq \max \mu_{d_{\ell}}$ and this also implies that $1-\max \left(\mu_{d_{\ell}}^{q}\right) \leq$ $1-\mu_{d_{\ell}}^{q} \leq 1-\min \left(\mu_{d_{\ell}}^{q}\right), \Leftrightarrow \prod_{\ell=1}^{n}\left(1-\max \left(\mu_{d_{\ell}}^{q}\right)\right)^{u_{\ell}} \leq$ $\prod_{\ell=1}^{n}\left(1-\mu_{d_{\ell}}^{q}\right)^{u_{\ell}} \leq \prod_{\ell=1}^{n}\left(1-\min \left(\mu_{d_{\ell}}^{q}\right)\right)^{u_{\ell}} \Leftrightarrow(1-$ $\left.\max \left(\mu_{d_{\ell}}^{q}\right)\right)^{\sum_{\ell=1}^{n} u_{\ell}} \leq \prod_{\ell=1}^{n}\left(1-\mu_{d_{\ell}}^{q}\right)^{u_{\ell}} \leq\left(1-\min \left(\mu_{d_{\ell}}^{q}\right)\right)^{\sum_{\ell=1}^{n} u_{\ell}}$

$\Leftrightarrow\left(1-\max \left(\mu_{d_{\ell}}^{q}\right)\right) \leq \prod_{\ell=1}^{n}\left(1-\mu_{d_{\ell}}^{q}\right)^{u_{\ell}} \leq\left(1-\min \left(\mu_{d_{\ell}}^{q}\right)\right)$

$\Leftrightarrow 1-\left(1-\min \left(\mu_{d_{\ell}}^{q}\right)\right) \leq 1-\prod_{\ell=1}^{n}\left(1-\mu_{d_{\ell}}^{q}\right)^{u_{\ell}} \leq 1-$ $\left(1-\max \left(\mu_{d_{\ell}}^{q}\right)\right)$

$\Leftrightarrow \min \left(\mu_{d_{\ell}}\right) \leq \sqrt[q]{1-\prod_{\ell=1}^{n}\left(1-\mu_{d_{\ell}}^{q}\right)^{u_{\ell}}} \leq \max \left(\mu_{d_{\ell}}\right)$

As $\hbar=\left(\mu_{\hbar}, \eta_{\hbar}\right)$ is a generalized parameter where $\mu_{\hbar}, \eta_{\hbar} \in[0,1]$, then

$$
\begin{gathered}
\mu_{\hbar}\left(\min \mu_{d_{\ell}}\right) \leq \mu_{\hbar} \cdot \sqrt[q]{1-\prod_{\ell=1}^{n}\left(1-\mu_{d_{\ell}}^{q}\right)^{u_{\ell}}} \leq \mu_{\hbar}\left(\max \mu_{d_{\ell}}\right) \\
\Leftrightarrow \min \mu_{\hbar \otimes d_{\ell}} \leq \mu_{\hbar} \cdot \sqrt[q]{1-\prod_{\ell=1}^{n}\left(1-\mu_{d_{\ell}}^{q}\right)^{u_{\ell}}} \leq \max \mu_{\hbar \otimes d_{\ell}}
\end{gathered}
$$

Next for each $\ell=1,2,3, \ldots, n$, we have $\min \eta_{d_{\ell}} \leq \eta_{d_{\ell}} \leq$ $\max \eta_{d_{\ell}}$ and this also implies that

$$
\begin{aligned}
& \prod_{\ell=1}^{n}\left(\left(\min \eta_{d_{\ell}}\right)^{q}\right)^{u_{\ell}} \\
& \quad \leq \prod_{\ell=1}^{n}\left(\eta_{d_{\ell}}^{u_{\ell}}\right)^{q} \leq \prod_{\ell=1}^{n}\left(\left(\max \eta_{d_{\ell}}\right)^{q}\right)^{u_{\ell}} \\
& \quad \Leftrightarrow\left(\left(\min \eta_{d_{\ell}}\right)^{q}\right)^{\sum_{\ell=1}^{n} u_{\ell}} \leq \prod_{\ell=1}^{n}\left(\eta_{d_{\ell}}^{u_{\ell}}\right)^{q} \leq\left(\left(\max \eta_{d_{\ell}}\right)^{q}\right)^{\sum_{\ell=1}^{n} u_{\ell}} \\
& \quad \Leftrightarrow\left(\min \eta_{d_{\ell}}\right)^{q} \leq \prod_{\ell=1}^{n}\left(\eta_{d_{\ell}}^{u_{\ell}}\right)^{q} \leq\left(\max \eta_{d_{\ell}}\right)^{q}
\end{aligned}
$$

As $\hbar=\left(\mu_{\hbar}, \eta_{\hbar}\right)$ is a generalized parameter where $\mu_{\hbar}, \eta_{\hbar} \in$ $[0,1]$, then

$$
\begin{aligned}
& \left(1-\eta_{\hbar}^{q}\right)\left(\min \eta_{d_{\ell}}\right)^{q} \\
& \leq\left(1-\eta_{\hbar}^{q}\right) \prod_{\ell=1}^{n}\left(\eta_{d_{\ell}}^{u_{\ell}}\right)^{q} \leq\left(1-\eta_{\hbar}^{q}\right)\left(\max \eta_{d_{\ell}}\right)^{q} \\
& \Leftrightarrow \eta_{\hbar}^{q}+\left(1-\eta_{\hbar}^{q}\right)\left(\min \eta_{d_{\ell}}\right)^{q} \\
& \leq \eta_{\hbar}^{q}+\left(1-\eta_{\hbar}^{q}\right) \prod_{\ell=1}^{n}\left(\eta_{d_{\ell}}^{u_{\ell}}\right)^{q} \leq \eta_{\hbar}^{q}+\left(1-\eta_{\hbar}^{q}\right)\left(\max \eta_{d_{\ell}}\right)^{q} \\
& \Leftrightarrow \sqrt[q]{\eta_{\hbar}^{q}+\left(1-\eta_{\hbar}^{q}\right)\left(\min \eta_{d_{\ell}}\right)^{q}} \\
& \leq \sqrt[q]{\eta_{\hbar}^{q}+\left(1-\eta_{\hbar}^{q}\right) \prod_{\ell=1}^{n}\left(\eta_{d_{\ell}}^{u_{\ell}}\right)^{q}} \\
& \leq \sqrt[q]{\eta_{\hbar}^{q}+\left(1-\eta_{\hbar}^{q}\right)\left(\max \eta_{d_{\ell}}\right)^{q}} \\
& \Leftrightarrow \sqrt[m i n]{\min \eta_{\hbar}} \\
& \leq \sqrt[q]{\eta_{\hbar}^{q}+\left(1-\eta_{\hbar}^{q}\right) \prod_{\ell=1}^{n}\left(\eta_{d_{\ell}}^{u_{\ell}}\right)^{q}} \leq \max \eta_{\hbar \otimes d_{\ell}}
\end{aligned}
$$

If $\mathrm{Gq}-\mathcal{R} O \mathcal{F} \mathcal{W} A\left(<d_{1}, d_{2}, d_{3}, \ldots, d_{n}>, \hbar\right)=\delta=$

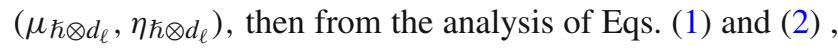
we have

$\min \mu_{\hbar \otimes d_{\ell}} \leq \mu_{\hbar \otimes d_{\ell}} \leq \max \mu_{\hbar \otimes d_{\ell}}$ and $\min \eta_{\hbar \otimes d_{\ell}} \leq$ $\eta_{\hbar \otimes d_{\ell}} \leq \max \eta_{\hbar \otimes d_{\ell}}$. Therefore, by score function, we get

$d_{\ell}^{-} \leq \mathrm{Gq}-\mathcal{R} O \mathcal{F} \mathcal{W} A\left(<d_{1}, d_{2}, d_{3}, \ldots, d_{n}>, \hbar\right) \leq d_{\ell}^{+}$

iii: Since $\mu_{d_{\ell}} \leq \mu_{d_{\ell}^{*}}$ and $\eta_{d_{\ell}} \geq \eta_{d_{\ell}^{*}}$ for all $\ell$, then this implies

$$
\begin{aligned}
& \mu_{d_{\ell}} \leq \mu_{d_{\ell}^{*}} \Rightarrow 1-\mu_{d_{\ell}^{*}}^{q} \leq 1-\mu_{d_{\ell}}^{q} \\
& \Rightarrow \prod_{\ell=1}^{n}\left(1-\mu_{d_{\ell}^{*}}^{q}\right)^{u_{\ell}} \leq \prod_{\ell=1}^{n}\left(1-\mu_{d_{\ell}}^{q}\right)^{u_{\ell}} \Rightarrow \\
& 1-\prod_{\ell=1}^{n}\left(1-\mu_{d_{\ell}}^{q}\right)^{u_{\ell}} \leq 1-\prod_{\ell=1}^{n}\left(1-\mu_{d_{\ell}^{*}}^{q}\right)^{u_{\ell}}
\end{aligned}
$$


$\Rightarrow \sqrt[q]{1-\prod_{\ell=1}^{n}\left(1-\mu_{d_{\ell}}^{q}\right)^{u_{\ell}}} \leq \sqrt[q]{1-\prod_{\ell=1}^{n}\left(1-\mu_{d_{\ell}^{*}}^{q}\right)^{u_{\ell}}}$

As $\hbar=\left(\mu_{\hbar}, \eta_{\hbar}\right)$ is a generalized parameter where $\mu_{\hbar}, \eta_{\hbar} \in$ $[0,1]$, then

$\mu_{\hbar} \cdot \sqrt[q]{1-\prod_{\ell=1}^{n}\left(1-\mu_{d_{\ell}}^{q}\right)^{u_{\ell}}} \leq \mu_{\hbar} \cdot \sqrt[q]{1-\prod_{\ell=1}^{n}\left(1-\mu_{d_{\ell}^{*}}^{q}\right)^{u_{\ell}}}$

Next

$$
\begin{aligned}
& \eta_{d_{\ell}} \geq \eta_{d_{\ell}^{*}} \Rightarrow \prod_{\ell=1}^{n}\left(\eta_{d_{\ell}}^{u_{\ell}}\right)^{q} \geq \prod_{\ell=1}^{n}\left(\eta_{d_{\ell}^{*}}^{u_{\ell}}\right)^{q} \\
& \Rightarrow\left(1-\eta_{\hbar}^{q}\right) \prod_{\ell=1}^{n}\left(\eta_{d_{\ell}}^{u_{\ell}}\right)^{q} \geq\left(1-\eta_{\hbar}^{q}\right) \prod_{\ell=1}^{n}\left(\eta_{d_{\ell}^{*}}^{u_{\ell}}\right)^{q} \\
& \Rightarrow \eta_{\hbar}^{q}+\left(1-\eta_{\hbar}^{q}\right) \prod_{\ell=1}^{n}\left(\eta_{d_{\ell}}^{u_{\ell}}\right)^{q} \geq \eta_{\hbar}^{q} \\
& +\left(1-\eta_{\hbar}^{q}\right) \prod_{\ell=1}^{n}\left(\eta_{d_{\ell}^{*}}^{u_{\ell}}\right)^{q} \\
& \Rightarrow \sqrt[q]{\eta_{\hbar}^{q}+\left(1-\eta_{\hbar}^{q}\right) \prod_{\ell=1}^{n}\left(\eta_{d_{\ell}}^{u_{\ell}}\right)^{q}} \\
& \geq \sqrt[q]{\eta_{\hbar}^{q}+\left(1-\eta_{\hbar}^{q}\right) \prod_{\ell=1}^{n}\left(\eta_{d_{\ell}^{*}}^{u_{\ell}}\right)^{q}}
\end{aligned}
$$

Let Gq-ROFW $O \mathcal{F}\left(<d_{1}, d_{2}, d_{3}, \ldots, d_{n}>, \hbar\right)=d$ and $\operatorname{Gq}-\mathcal{R} O \mathcal{F} \mathcal{W} A\left(<d_{1}^{*}, d_{2}^{*}, d_{3}^{*}, \ldots, d_{n}^{*}>, \hbar\right)=d^{*}$. Now by Eqs. (3), (4) and Part i: (idempotency), we have

$$
\begin{aligned}
& \left(\mu_{\hbar}\right)^{q} \cdot\left(1-\prod_{\ell=1}^{n}\left(1-\mu_{d_{\ell}^{*}}^{q}\right)^{u_{\ell}}\right) \\
& -\left(\eta_{\hbar}^{q}+\left(1-\eta_{\hbar}^{q}\right) \prod_{\ell=1}^{n}\left(\eta_{d_{\ell}^{*}}^{u_{\ell}}\right)^{q}\right) \\
& \leq\left(\mu_{\hbar}\right)^{q} \cdot\left(1-\prod_{\ell=1}^{n}\left(1-\mu_{d_{\ell}}^{q}\right)^{u_{\ell}}\right) \\
& \quad-\left(\eta_{\hbar}^{q}+\left(1-\eta_{\hbar}^{q}\right) \prod_{\ell=1}^{n}\left(\eta_{d_{\ell}}^{u_{\ell}}\right)^{q}\right) \\
& \Rightarrow S\left(d^{*}\right) \geq S(d)
\end{aligned}
$$

If $S\left(d^{*}\right)>S(d)$, then

$$
\begin{aligned}
& \operatorname{Gq}-\mathcal{R} O \mathcal{F} \mathcal{W} A\left(<d_{1}^{*}, d_{2}^{*}, d_{3}^{*}, \ldots, d_{n}^{*}>\right. \\
& \hbar)>\operatorname{Gq}-\mathcal{R} O \mathcal{F W} A\left(<d_{1}, d_{2}, d_{3}, \ldots, d_{n}>, \hbar\right)
\end{aligned}
$$

If $S\left(d^{*}\right)=S(d)$, then $\left(\mu_{\hbar}\right)^{q} \cdot\left(1-\prod_{\ell=1}^{n}\left(1-\mu_{d_{\ell}^{*}}^{q}\right)^{u_{\ell}}\right)-\left(\eta_{\hbar}^{q}+\left(1-\eta_{\hbar}^{q}\right) \prod_{\ell=1}^{n}\left(\eta_{d_{\ell}^{*}}^{u_{\ell}}\right)^{q}\right)$

$=\left(\mu_{\hbar}\right)^{q} \cdot\left(1-\prod_{\ell=1}^{n}\left(1-\mu_{d_{\ell}}^{q}\right)^{u_{\ell}}\right)$

$-\left(\eta_{\hbar}^{q}+\left(1-\eta_{\hbar}^{q}\right) \prod_{\ell=1}^{n}\left(\eta_{d_{\ell}}^{u_{\ell}}\right)^{q}\right)$. Since $\mu_{d_{\ell}} \leq \mu_{d_{\ell}^{*}}$ and $\eta_{d_{\ell}} \geq$ $\eta_{d_{\ell}^{*}}$ for all $\ell$, so we have

$\Rightarrow\left(\mu_{\hbar}\right)^{q} \cdot\left(1-\prod_{\ell=1}^{n}\left(1-\mu_{d_{\ell}^{*}}^{q}\right)^{u_{\ell}}\right)$

$=\left(\mu_{\hbar}\right)^{q} \cdot\left(1-\prod_{\ell=1}^{n}\left(1-\mu_{d_{\ell}}^{q}\right)^{u_{\ell}}\right)$ and

$\left(\eta_{\hbar}^{q}+\left(1-\eta_{\hbar}^{q}\right) \prod_{\ell=1}^{n}\left(\eta_{d_{\ell}^{*}}^{u_{\ell}}\right)^{q}\right)=\left(\eta_{\hbar}^{q}+\left(1-\eta_{\hbar}^{q}\right) \prod_{\ell=1}^{n}\left(\eta_{d_{\ell}}^{u_{\ell}}\right)^{q}\right)$.

Thus by accuracy function

$A\left(d^{*}\right)=A(d)$

Therefore, from the above analysis, we have

$$
\begin{array}{r}
\operatorname{Gq}-\mathcal{R} O \mathcal{F} \mathcal{W} A\left(<d_{1}, d_{2}, d_{3}, \ldots, d_{n}>, \hbar\right) \geq \\
\operatorname{Gq}-\mathcal{R} O \mathcal{F} \mathcal{W} A\left(<d_{1}^{*}, d_{2}^{*}, d_{3}^{*}, \ldots, d_{n}^{*}>, \hbar\right)
\end{array}
$$

iv: Proof is straightforward and a trivial case of Theorem 4.

If it is suppose that the experts have enough knowledge about the evaluated objects and assign value to generalized parameter $\hbar=(1,0)$. In this case, the proposed Gq$\mathcal{R} O \mathcal{F} \mathcal{W} A$ operator reduces to $q-\mathcal{R} O \mathcal{F} \mathcal{W} A$ operator. While these types of situations are partially fulfilled in real life. This shortcoming is very carefully tackled by adding the evaluated object/generalized parameter in the initial given preferences.

Proposition 1 (a) If the priority of the senior decision makerlexpert about generalized parameter is taken as $\hbar=$ $(1,0)$, then the proposed $G q-\mathcal{R} O \mathcal{F} \mathcal{W} A$ operator reduces to $q$ - $\mathcal{R} O \mathcal{F} \mathcal{W}$ A operator.

(b) If the priority of the senior decision maker/expert about generalized parameter is taken as $\hbar=(0,1)$, then the proposed $G q-\mathcal{R} O \mathcal{F} \mathcal{W}$ A operator gives the result $(0,1)$.

Proof (a) If it is assumed that the experts have enough knowledge about the evaluated objects, that is the generalized parameter $\hbar=(1,0)$, then in Theorem 4 , we have

$$
\begin{aligned}
\mathrm{Gq} & -\mathcal{R} O \mathcal{F} \mathcal{W} A\left(<d_{1}, d_{2}, d_{3}, \ldots, d_{n}>, \hbar\right) \\
= & \left(\mu_{\hbar} \cdot \sqrt[q]{1-\prod_{\ell=1}^{n}\left(1-\mu_{d_{\ell}}^{q}\right)^{u_{\ell}}}\right. \\
& \sqrt[q]{\left.\eta_{\hbar}^{q}+\left(1-\eta_{\hbar}^{q}\right) \prod_{\ell=1}^{n}\left(\eta_{d_{\ell}}^{u_{\ell}}\right)^{q}\right)}
\end{aligned}
$$


$=\left(\sqrt[q]{1-\prod_{\ell=1}^{n}\left(1-\mu_{d_{\ell}}^{q}\right)^{u_{\ell}}}, \sqrt[q]{\prod_{\ell=1}^{n}\left(\eta_{d_{\ell}}^{u_{\ell}}\right)^{q}}\right)$

as $\mu_{\hbar}=1$ and $\eta_{\hbar}=0$

$$
\begin{aligned}
& =\left(\sqrt[q]{1-\prod_{\ell=1}^{n}\left(1-\mu_{d_{\ell}}^{q}\right)^{u_{\ell}}, \prod_{\ell=1}^{n} \eta_{d_{\ell}}^{u_{\ell}}}\right) \\
& =q-\mathcal{R} O \mathcal{F} \mathcal{W} A\left(d_{1}, d_{2}, d_{3}, \ldots, d_{n}\right) .
\end{aligned}
$$

(b) The proof is similar to the Proof of $(a)$.

\section{The generalized q-rung orthopair fuzzy ordered weighted average operator $(\mathrm{Gq}-\mathcal{R} 0 \mathcal{F} O \mathcal{W A})$}

From $\mathrm{Gq}-\mathcal{R} \mathrm{O} \mathcal{F} \mathrm{WA}$, it is clear that in $\mathrm{Gq}-\mathcal{R} \mathrm{O} \mathcal{F}$ WA operators just the $\mathrm{q}-\mathcal{R O} \mathcal{F}$ values are weighed on the basis of generalized parameter, and the $\mathrm{Gq}-\mathcal{R} \mathrm{O} \mathcal{F} \mathrm{OWA}$ operator weight the ordered positions through scoring the $\mathrm{q}-\mathcal{R} \mathrm{O} \mathcal{F}$ values rather than weighting the $\mathrm{q}-\mathcal{R O} \mathcal{F}$ values themselves on the basis of generalized parameter. Therefore, here, we will present the detailed study of $\mathrm{Gq}-\mathcal{R} \mathrm{O} \mathcal{F} \mathrm{O} \mathcal{W}$ A operator and their properties.

Definition 10 Consider a generalized parameter $\hbar=\left(\mu_{\hbar}, \eta_{\hbar}\right)$ for the q- $\mathcal{R O} \mathcal{F} \mathrm{Ns} d_{\ell}=\left(\mu_{d_{\ell}}, \eta_{d_{\ell}}\right)$ (for $\ell=1,2, \ldots, n$ ), then the $\mathrm{Gq}-\mathcal{R} \mathrm{O} \mathcal{F} O \mathcal{W}$ A operator is given as

$$
\begin{array}{r}
\operatorname{Gq}-\mathcal{R} O \mathcal{F} O \mathcal{W} A\left(<d_{1}, d_{2}, d_{3}, \ldots, d_{n}>, \hbar\right) \\
=\hbar \otimes q-\mathcal{R O F} O \mathcal{W} A\left(\tilde{d}_{1}, \tilde{d}_{2}, \tilde{d}_{3}, \ldots, \tilde{d}_{n}\right) .
\end{array}
$$

The aggregation result of Definition 10 through operation rules is described as in Theorem 6 .

Theorem 6 Suppose the collection $d_{\ell}=\left(\mu_{d_{\ell}}, \eta_{d_{\ell}}\right)(\ell=$ $1,2, \ldots, n)$ of $q$ - $\mathcal{R} O \mathcal{F} N s$ with generalized parameter $\hbar=$ $\left(\mu_{\hbar}, \eta_{\hbar}\right)$ and weight vector $u=\left(u_{1}, u_{2}, \ldots, u_{n}\right)^{\mathrm{T}}$ of $d_{\ell}$ where $u_{\ell} \in[0,1]$ such that $\sum_{\ell=1}^{n} u_{\ell}=1$, then the Gq$\mathcal{R O F} O \mathcal{W} A$ operator is described as:

$$
\begin{aligned}
& G q-\mathcal{R O F} O \mathcal{W} A\left(<d_{1}, d_{2}, d_{3}, \ldots, d_{n}>, \hbar\right) \\
& =\hbar \otimes\left(\stackrel{n}{\ell=1}_{\ell}^{n} u_{\ell} \tilde{d}_{\ell}\right) \\
& =\left(\mu_{\hbar} \cdot \sqrt[q]{1-\prod_{\ell=1}^{n}\left(1-\mu_{\tilde{d}_{\ell}}^{q}\right)^{u_{\ell}}}, \sqrt[q]{\left.\eta_{\hbar}^{q}+\left(1-\eta_{\hbar}^{q}\right) \prod_{\ell=1}^{n}\left(\eta_{\tilde{d}_{\ell}}^{u_{\ell}}\right)^{q}\right)}\right.
\end{aligned}
$$

where $\tilde{d}_{\ell}=\left(\mu_{\tilde{d}_{\ell}}, \eta_{\tilde{d}_{\ell}}\right)$ indicate the permutation of $\ell$ th largest object of the collection of $n q$-ROF $O \mathcal{F} s d_{\ell}=\left(\mu_{d_{\ell}}, \eta_{d_{\ell}}\right)(\ell=$ $1,2, \ldots, n)$.

Proof The proof is straightforward to Theorem 4.
Remark 2 (a) If the generalized parameter $\hbar=(1,0)$, and $q=1$, then the $\mathrm{Gq}-\mathcal{R} \mathrm{O} \mathcal{F} \mathrm{O} \mathcal{W} \mathrm{A}$ operator reduces to I $\mathcal{F O} \mathcal{W}$ A operator.

(b) If the generalized parameter $\hbar=(1,0)$, and $q=$

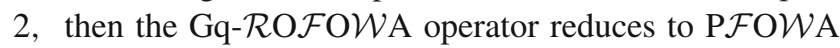
operator.

(c) If the value of $q=2$ is fixed then the $\mathrm{Gq}-\mathcal{R O} \mathcal{F O} \mathcal{W A}$ operator reduces to $\mathrm{GP} \mathcal{F} \mathrm{O} \mathcal{W} A$ operator.

Example 5 Suppose the generalized parameter $\hbar=$ $(0.95,0.7)$ of four $q-\mathcal{R} O \mathcal{F} N s d_{1}=(0.7,0.3), d_{2}=$ $(0.8,0.65), d_{3}=(0.9,0.6), d_{4}=(0.88,0.7)$ having weight vector $u=\{0.3,0.25,0.1,0.35\}$ for $q=5$, then to find the score functions of each $\mathrm{q}-\mathcal{R} \mathrm{O} \mathcal{F} \mathrm{Ns}$, that is

$S\left(d_{1}\right)=0.7^{5}-0.3^{5}=0.166, S\left(d_{2}\right)=0.8^{5}-0.65^{5}=$ $0.212, S\left(d_{3}\right)=0.9^{5}-0.6^{5}=0.513, S\left(d_{4}\right)=0.88^{5}-$ $0.7^{5}=0.360$.

So $S\left(d_{3}\right)>S\left(d_{4}\right)>S\left(d_{2}\right)>S\left(d_{1}\right)$, this implies that $d_{4}>d_{3}>d_{2}>d_{1}$. Thus $\tilde{d}_{1}=d_{3}, \tilde{d}_{2}=d_{4}, \tilde{d}_{3}=d_{2}$, and $\tilde{d}_{4}=d_{1}$. Further we have

$$
\begin{aligned}
& \mu_{\hbar} \cdot \sqrt[5]{1-\prod_{\ell=1}^{4}\left(1-\mu_{\tilde{d}_{\ell}}^{5}\right)^{u_{\ell}}}=0.95 \times \\
& \sqrt[5]{\frac{1-\left(1-0.9^{5}\right)^{0.3}\left(1-0.88^{5}\right)^{0.25}\left(1-0.8^{5}\right)^{0.1}}{\left(1-0.7^{5}\right)^{0.35}}}=0.802 \\
& \sqrt[5]{\eta_{\hbar}^{5}+\left(1-\eta_{\hbar}^{5}\right) \prod_{\ell=1}^{4}\left(\eta_{\tilde{d}_{\ell}}^{u_{\ell}}\right)^{5}}= \\
& \sqrt[5]{0.7^{5}+\left(1-0.7^{5}\right)\left(0.6^{0.3}\right)^{5}\left(0.7^{0.25}\right)^{5}\left(0.65^{0.1}\right)^{5}\left(0.3^{0.35}\right)^{5}}=0.720
\end{aligned}
$$

Now by Theorem 6 , we have

$$
\begin{aligned}
& \operatorname{Gq}-\mathcal{R} O \mathcal{F} O \mathcal{W} A\left(<d_{1}, d_{2}, d_{3}, d_{4}>\hbar\right) \\
& =\left(\mu_{\hbar} \cdot \sqrt[5]{1-\prod_{\ell=1}^{4}\left(1-\mu_{\tilde{d}_{\ell}}^{5}\right)^{u_{\ell}}}, \sqrt[5]{\eta_{\hbar}^{5}+\left(1-\eta_{\hbar}^{5}\right) \prod_{\ell=1}^{4}\left(\eta_{\tilde{d}_{\ell}}^{u_{\ell}}\right)^{5}}\right) \\
& =(0.802,0.720)
\end{aligned}
$$

Theorem 7 Suppose the collection $d_{\ell}=\left(\mu_{d_{\ell}}, \eta_{d_{\ell}}\right)(\ell=$ $1,2, \ldots, n)$ of $q$-R $O \mathcal{F} N$ s with generalized parameter $\hbar=$ $\left(\mu_{\hbar}, \eta_{\hbar}\right)$ and associated weight vector $u=\left(u_{1}, u_{2}, \ldots, u_{n}\right)^{\mathrm{T}}$ of $d_{\ell}$ where $u_{\ell} \in[0,1]$ such that $\sum_{\ell=1}^{n} u_{\ell}=1$, then for $G q$ ROF $O W$ A operator the following are holes:

i: (Idempotency): If $\tilde{d}_{\ell}=\tilde{d}$ (for all $\left.\ell=1,2,3, \ldots, n\right)$, then

$G q-\mathcal{R O F} O \mathcal{W} A\left(<d_{1}, d_{2}, d_{3}, \ldots, d_{n}>, \hbar\right)=\hbar \otimes \tilde{d}$.

ii: (Boundary condition): If $\tilde{d}_{\ell}^{-}=\left(\min \mu_{\hbar \otimes \tilde{d}_{\ell}}, \max \eta_{\hbar \otimes \tilde{d}_{\ell}}\right)$ and $\tilde{d}_{\ell}^{+}=\left(\max \mu_{\hbar \otimes \tilde{d}_{\ell}}, \min \eta_{\hbar \otimes \tilde{d}_{\ell}}\right)$ (for all $\ell=$ $1,2,3, \ldots, n)$, then

$\tilde{d}_{\ell}^{-} \leq G q-\mathcal{R O F} O \mathcal{W} A\left(<d_{1}, d_{2}, d_{3}, \ldots, d_{n}>, \hbar\right) \leq \tilde{d}_{\ell}^{+}$. 
iii: (Monotonicity): Suppose $d_{\ell}=\left(\mu_{d_{\ell}}, \eta_{d_{\ell}}\right)$ and $d_{\ell}^{*}=$ $\left(\mu_{d_{\ell}^{*}}, \eta_{d_{\ell}^{*}}\right)(\ell=1,2, \ldots, n)$ be the collection of $n q-$ ROFNs such that $\mu_{d_{\ell}} \leq \mu_{d_{\ell}^{*}}$ and $\eta_{d_{\ell}} \geq \eta_{d_{\ell}^{*}}$, then

$$
\begin{array}{r}
G q-\mathcal{R} O \mathcal{F} O \mathcal{W} A\left(<d_{1}, d_{2}, d_{3}, \ldots, d_{n}>, \hbar\right) \leq \\
G q-\mathcal{R O F} O \mathcal{W} A\left(<d_{1}^{*}, d_{2}^{*}, d_{3}^{*}, \ldots, d_{n}^{*}>, \hbar\right) .
\end{array}
$$

iv: (Commutativity): Suppose $d_{\ell}=\left(\mu_{d_{\ell}}, \eta_{d_{\ell}}\right)$ and $\tilde{d}_{\ell}=$ $\left(\mu_{\tilde{d}_{\ell}}, \eta_{\tilde{d}_{\ell}}\right)(\ell=1,2, \ldots, n)$ be the collection of $n q-$ ROFNs where $\tilde{d}_{\ell}(\ell=1,2, \ldots, n)$ is any permutation of $d_{\ell}(\ell=1,2, \ldots, n)$, then

$$
\begin{aligned}
& G q-\mathcal{R O F} O \mathcal{W} A\left(<d_{1}, d_{2}, d_{3}, \ldots, d_{n}>, \hbar\right) \\
& \quad=G q-\mathcal{R O F} O \mathcal{W} A\left(<\tilde{d}_{1}, \tilde{d}_{2}, \tilde{d}_{3}, \ldots, \tilde{d}_{n}>, \hbar\right)
\end{aligned}
$$

Proof Proofs are easy and can be done from Theorem 5.

Proposition 2 (a) If the priority of the senior decision maker/expert about generalized parameter is taken as $\hbar=$ $(1,0)$, so in this case the proposed $G q-\mathcal{R} O \mathcal{F} O \mathcal{W} A$ operator degenerates to $q-\mathcal{R} O \mathcal{F} O \mathcal{W}$ A operator.

(b) If the priority of the senior decision maker/expert about generalized parameter is taken as $\hbar=(0,1)$, so in this case the proposed $G q-\mathcal{R} O \mathcal{F} O \mathcal{W}$ A operator gives the result $(0,1)$.

Proof The proofs are similar to the Proposition 1.

\section{The generalized q-rung orthopair fuzzy hybrid aggregation $(G q-\mathcal{R} O \mathcal{F} H A)$ operator}

From the detail discussion of Gq- $\mathcal{R} O \mathcal{F} W A$ and Gq$\mathcal{R O} \mathcal{F O W A}$ operators, it is clear that in Gq- $\mathcal{R}$ OFWA operators just the $\mathrm{q}-\mathcal{R O} \mathcal{F}$ values are weighed on the basis of generalized parameter, and similarly in Gq- $\mathcal{R} O \mathcal{F} O W A$ operator just the ordered positions of the $\mathrm{q}-\mathcal{R} O \mathcal{F}$ values are weighed rather than weighting the $\mathrm{q}-\mathcal{R}$ OF $\mathcal{F}$ values themselves on the base of generalized parameter. So, it is clear that the weights denote distinct attributes in both Gq$\mathcal{R O} \mathcal{F W A}$ and Gq- $\mathcal{R} O \mathcal{F}$ OWA operators. However, at the same time both the operators weigh just one of them. To handle this restriction, here we will originate the concept of $\mathrm{Gq}-\mathcal{R} \mathrm{O} \mathcal{F}$ HA operator, which weighs both the given values at the same time, that is $\mathrm{q}-\mathcal{R} O \mathcal{F}$ values and its ordered position on the basis of generalized parameter and discuss their properties in detail.

Definition 11 Consider $d_{\ell}=\left(\mu_{d_{\ell}}, \eta_{d_{\ell}}\right)($ for $\ell=1,2, \ldots, n)$ be the collections of $\mathrm{q}-\mathcal{R} O \mathcal{F}$ Ns with associated weight vector $u=\left(u_{1}, u_{2}, \ldots, u_{n}\right)^{\mathrm{T}}$ of $d_{\ell}$ where $u_{\ell} \in[0,1]$ such that $\sum_{\ell=1}^{n} u_{\ell}=1$, under generalized parameter $\hbar=\left(\mu_{\hbar}, \eta_{\hbar}\right)$ and $\mathfrak{u}=\left({\mathfrak{u}_{1}}_{1}, \text { ü }_{2}, \ldots, \text { ü }_{n}\right)^{\mathrm{T}}$ be the weight vector of $d_{\ell}(\ell=$
$1,2, \ldots, n)$ where $\tilde{u}_{\ell} \in[0,1]$ such that $\sum_{\ell=1}^{n} \tilde{u}_{\ell}=1$. Then, the $\mathrm{Gq}-\mathcal{R O} \mathcal{F} H A$ operator is given as:

$$
\begin{gathered}
\mathrm{Gq}-\mathcal{R} O \mathcal{F} H A\left(<d_{1}, d_{2}, d_{3}, \ldots, d_{n}>, \hbar\right) \\
=\hbar \otimes q-\mathcal{R} O \mathcal{F} H A\left(\tilde{d}_{1}, \tilde{d}_{2}, \tilde{d}_{3}, \ldots, \tilde{d}_{n}\right) .
\end{gathered}
$$

The aggregation result of Definition 11 through operation rules is described as in Theorem 8 .

Theorem 8 Consider a generalized parameter $\hbar=\left(\mu_{\hbar}, \eta_{\hbar}\right)$ for the $q$ - $\mathcal{R} O \mathcal{F} N s d_{\ell}=\left(\mu_{d_{\ell}}, \eta_{d_{\ell}}\right)($ for $\ell=1,2, \ldots, n)$, then the $G q-\mathcal{R} O \mathcal{F} H A$ operator is described as:

$$
\begin{aligned}
& G q-\mathcal{R} O \mathcal{F} H A\left(<d_{1}, d_{2}, d_{3}, \ldots, d_{n}>, \hbar\right)=\hbar \otimes\left(\bigoplus_{\ell=1}^{n} u_{\ell} \tilde{d}_{\ell}\right) \\
& =\left(\mu_{\hbar} \cdot \sqrt[q]{1-\prod_{\ell=1}^{n}\left(1-\mu_{\tilde{d}_{\ell}}^{q}\right)^{u_{\ell}}}, \sqrt[q]{\eta_{\hbar}^{q}+\left(1-\eta_{\hbar}^{q}\right) \prod_{\ell=1}^{n}\left(\eta_{\tilde{d}_{\ell}}^{u_{\ell}}\right)^{q}}\right)
\end{aligned}
$$

where $\tilde{d}_{\ell}\left(\tilde{d}_{\ell}=n u_{\ell} d_{\ell}\right.$, for $\left.\ell=1,2, \ldots, n\right)$ indicate the permutation of $\ell$ th largest object of the collection of $q-\mathcal{R} O \mathcal{F} N s$ $d_{\ell}=\left(\mu_{d_{\ell}}, \eta_{d_{\ell}}\right)(\ell=1,2, \ldots, n)$ such that $n$ indicate the balancing coefficient.

Proof Straightforward like Theorem 4.

Remark 3 (a) If the generalized parameter $\hbar=(1,0)$, and $q=1$, so in this case $\mathrm{Gq}-\mathcal{R} \mathrm{O} \mathcal{F} H A$ operator degenerates to I $\mathcal{F H A}$ operator.

(b) If the generalized parameter $\hbar=(1,0)$, and $q=2$, so in this case $\mathrm{Gq}-\mathcal{R} \mathrm{O} \mathcal{F} \mathrm{HA}$ operator degenerates to $\mathrm{P} \mathcal{F} H A$ operator. (c) If the value of parameter $q=2$ is fixed then the $\mathrm{Gq}-\mathcal{R O} \mathcal{F} H A$ operator reduces to GP $\mathcal{F} H A$ operator.

Example 6 Suppose the generalized parameter $\hbar=(0.8,0.7)$ of four $q$-R $O \mathcal{F} N s d_{1}=(0.5,0.2), d_{2}=(0.83,0.6)$, $d_{3}=(0.95,0.65), d_{4}=(0.9,0.75)$ having associated weight vector $u=\{0.3,0.2,0.32,0.18\}$ for $q=4$, and weight vector $u=\{0.4,0.3,0.1,0.2\}$, then using operational law

$n \tilde{u}_{\ell} d_{\ell}=\left(\sqrt[q]{1-\left(1-\mu_{d_{\ell}}^{q}\right)^{n u_{\ell}}}, \eta_{d_{\ell}}^{n \tilde{u}_{\ell}}\right)$

we have

$$
\begin{aligned}
4 \tilde{u}_{1} d_{1} & =\left(\sqrt[4]{1-\left(1-0.5^{4}\right)^{4 \times 0.4}}, 0.2^{4 \times 0.4}\right) \\
& =(0.560,0.076), \\
4 \tilde{u}_{2} d_{2} & =\left(\sqrt[4]{1-\left(1-0.83^{4}\right)^{4 \times 0.3}}, 0.6^{4 \times 0.3}\right) \\
& =(0.856,0.542), \\
4 \tilde{u}_{3} d_{3} & =\left(\sqrt[4]{1-\left(1-0.95^{4}\right)^{4 \times 0.1}}, 0.65^{4 \times 0.1}\right) \\
& =(0.837,0.842),
\end{aligned}
$$




$$
\begin{aligned}
\text { ǘ }_{4} d_{4} & =\left(\sqrt[4]{1-\left(1-0.9^{4}\right)^{4 \times 0.2}}, 0.75^{4 \times 0.2}\right) \\
& =(0.870,0.794) .
\end{aligned}
$$

Now to find their score functions, that is

$$
\begin{aligned}
S\left(4 u_{1} d_{1}\right) & =0.560^{4}-0.076^{4}=0.098, \\
S\left(4 u_{2} d_{2}\right) & =0.856^{4}-0.542^{4}=0.451, \\
S\left(4 u_{3} d_{3}\right) & =0.837^{4}-0.842^{4} \\
& =-0.012, \\
S\left(4 \tilde{u}_{4} d_{4}\right) & =0.0 .870^{4}-0.794^{4}=-0.396 .
\end{aligned}
$$

So $S\left(4 \tilde{u}_{2} d_{2}\right)>S\left(4 \tilde{u}_{1} d_{1}\right)>S\left(4 \tilde{u}_{3} d_{3}\right)>S\left(4 \tilde{u}{ }_{4} d_{4}\right)$, this implies that

$$
\begin{aligned}
& \tilde{d}_{1}=(0.856,0.542), \tilde{d}_{2}=(0.560,0.076), \\
& \tilde{d}_{3}=(0.837,0.842) \text { and } \tilde{d}_{4}=(0.870,0.794)
\end{aligned}
$$

Furthermore, we have $\mu_{\hbar} \cdot \sqrt[4]{1-\prod_{\ell=1}^{4}\left(1-\mu_{\tilde{d}_{\ell}}^{4}\right)^{u_{\ell}}}=0.8 \times$ $0.824648=0.65972$, and $\sqrt[4]{\eta_{\hbar}^{4}+\left(1-\eta_{\hbar}^{4}\right) \prod_{\ell=1}^{4}\left(\eta_{\tilde{d}_{\ell}}^{u_{\ell}}\right)^{4}}=$ 0.72192

Now by Theorem 8 , we have

$$
\begin{aligned}
& \text { Gq- } \mathcal{R} O \mathcal{F} H A\left(<d_{1}, d_{2}, d_{3}, d_{4}>, \hbar\right) \\
& =\left(\mu_{\hbar} \cdot \sqrt[4]{1-\prod_{\ell=1}^{4}\left(1-\mu_{\tilde{d}_{\ell}}^{4}\right)^{u_{\ell}}}, \sqrt[4]{\eta_{\hbar}^{4}+\left(1-\eta_{\hbar}^{4}\right) \prod_{\ell=1}^{4}\left(\eta_{\tilde{d}_{\ell}}^{u_{\ell}}\right)^{4}}\right) \\
& =(0.65972,0.72192)
\end{aligned}
$$

Theorem 9 Suppose the collection $d_{\ell}=\left(\mu_{d_{\ell}}, \eta_{d_{\ell}}\right)(\ell=$ $1,2, \ldots, n)$ of $q$-ROF $N$ s with associated weight vector $u=\left(u_{1}, u_{2}, \ldots, u_{n}\right)^{\mathrm{T}}$ of $d_{\ell}$ where $u_{\ell} \in[0,1]$ such that $\sum_{\ell=1}^{n} u_{\ell}=1$, on the base of generalized parameter $\hbar=$ $\left(\mu_{\hbar}, \eta_{\hbar}\right)$ and $\ddot{u}=\left(\ddot{u}_{1}, \ddot{u}_{2}, \ldots, \ddot{u}_{n}\right)^{\mathrm{T}}$ be the weight vector of $d_{\ell}(\ell=1,2, \ldots, n)$ where $\ddot{u}_{\ell} \in[0,1]$ such that $\sum_{\ell=1}^{n} \ddot{u}_{\ell}=1$ then for $G q-\mathcal{R} O \mathcal{F} H A$ operator the following assertions are hole:

i: (Idempotency): If $\tilde{d}_{\ell}=\tilde{d}($ for all $\ell=1,2,3, \ldots, n)$, then

$G q-\mathcal{R} O \mathcal{F} H A\left(<d_{1}, d_{2}, d_{3}, \ldots, d_{n}>, \hbar\right)=\hbar \otimes \tilde{d}$

ii: (Boundary condition): If $\tilde{d}_{\ell}^{-}=\left(\min \mu_{\hbar \otimes \tilde{d}_{\ell}}, \max \eta_{\hbar \otimes \tilde{d}_{\ell}}\right)$ and $\tilde{d}_{\ell}^{+}=\left(\max \mu_{\hbar \otimes \tilde{d}_{\ell}}, \min \eta_{\hbar \otimes \tilde{d}_{\ell}}\right)($ for all $\ell=$ $1,2,3, \ldots, n)$, then

$\tilde{d}_{\ell}^{-} \leq G q-\mathcal{R} O \mathcal{F} H A\left(<d_{1}, d_{2}, d_{3}, \ldots, d_{n}>, \hbar\right) \leq \tilde{d}_{\ell}^{+}$. iii: (Monotonicity): Suppose $d_{\ell}=\left(\mu_{d_{\ell}}, \eta_{d_{\ell}}\right)$ and $d_{\ell}^{*}=$ $\left(\mu_{d_{\ell}^{*}}, \eta_{d_{\ell}^{*}}\right)(\ell=1,2, \ldots, n)$ be two collections of $n q-$ $\mathcal{R} O \mathcal{F} N$ s in which $\mu_{d_{\ell}} \leq \mu_{d_{\ell}^{*}}$ and $\eta_{d_{\ell}} \geq \eta_{d_{\ell}^{*}}$, then

$$
\begin{aligned}
& G q-\mathcal{R} O \mathcal{F} H A\left(<d_{1}, d_{2}, d_{3}, \ldots, d_{n}>, \hbar\right) \\
& \quad \leq G q-\mathcal{R} O \mathcal{F} H A\left(<d_{1}^{*}, d_{2}^{*}, d_{3}^{*}, \ldots, d_{n}^{*}>, \hbar\right) .
\end{aligned}
$$

iv: (Commutativity): Suppose $d_{\ell}=\left(\mu_{d_{\ell}}, \eta_{d_{\ell}}\right)$ and $\tilde{d}_{\ell}=$ $\left(\mu_{\tilde{d}_{\ell}}, \eta_{\tilde{d}_{\ell}}\right)(\ell=1,2, \ldots, n)$ be two collection of $n q-$ $\mathcal{R} O \mathcal{F} N$ s in which $\tilde{d}_{\ell}(\ell=1,2, \ldots, n)$ is the lth largest object of $d_{\ell}(\ell=1,2, \ldots, n)$, then

$$
\begin{aligned}
& G q-\mathcal{R O} \mathcal{F} H A\left(<d_{1}, d_{2}, d_{3}, \ldots, d_{n}>, \hbar\right) \\
& \quad=G q-\mathcal{R O} O \mathcal{F} H A\left(<\tilde{d}_{1}, \tilde{d}_{2}, \tilde{d}_{3}, \ldots, \tilde{d}_{n}>, \hbar\right) .
\end{aligned}
$$

Proof Proofs are easy and can be done from Theorem 5.

Proposition 3 (a) If the priority of the senior decision maker/expert about generalized parameter is taken as $\hbar=$ $(1,0)$, then the proposed $G q-\mathcal{R} O \mathcal{F} H A$ operator reduces to $q-\mathcal{R} O \mathcal{F} H A$ operator.

(b) If the priority of the senior decision maker/expert about generalized parameter is taken as $\hbar=(0,1)$, so in this case the proposed $G q-\mathcal{R} O \mathcal{F} H A$ operator gives the result $(0,1)$.

Proof Proofs are straightforward.

Remark 4 (a) If $\tilde{u}=\left(\frac{1}{n}, \frac{1}{n}, \ldots, \frac{1}{n}\right)^{\mathrm{T}}$, then the proposed $G q$ $\mathcal{R} O \mathcal{F} H A$ operator reduces to $G q-\mathcal{R} O \mathcal{F} W A$ operator.

(b) If $u=\left(\frac{1}{n}, \frac{1}{n}, \ldots, \frac{1}{n}\right)^{\mathrm{T}}$, then the proposed $G q$ $\mathcal{R} O \mathcal{F} H A$ operator reduces to $G q-\mathcal{R} O \mathcal{F} O W A$ operator.

\section{Group generalized parameter based on q-rung orthopair fuzzy average aggregation operator}

In this section, we will present the generalized study of the proposed aggregation operators. This analysis will based on two or more expert's/observer's opinion in original information by combining the different choice and expertise of the senior decision makers/experts in a more accurate way. Therefore, this can be obtained by introducing a group Gq$\mathcal{R O} \mathcal{F} W A(\mathrm{GGq}-\mathcal{R} O \mathcal{F} \mathrm{WA})$ operator, group $\mathrm{Gq}-\mathcal{R} O \mathcal{F} O W A$

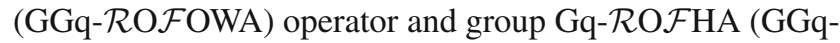
$\mathcal{R O} \mathcal{F}$ HA) operator.

\section{The GGq- $\mathcal{R} 0 \mathcal{F}$ WA operator}

In this subsection, the idea of generalized q- $\mathcal{R} O \mathcal{F} W A$ operator is explored to group generalized q- $\mathcal{R} O \mathcal{F} W A$ operator where the preferences of two or more other senior experts/decision makers are analyze in q-ROF environments. 
Definition 12 Consider a group of experts/observers who justify the information under the $\mathrm{q}-\mathcal{R O} \mathcal{F}$ environment. Let $\hbar_{k}=\left(\mu_{\hbar_{k}}, \eta_{\hbar_{k}}\right)(k=1,2, \ldots, m)$ be the preferences suggested by the senior experts for the q-ROFNs $d_{\ell}=$ $\left(\mu_{d_{\ell}}, \eta_{d_{\ell}}\right)(\ell=1,2, \ldots, n)$, then the GGq- $\mathcal{R} O \mathcal{F}$ WA operators is given as:

$$
\begin{aligned}
& \operatorname{GGq}-\mathcal{R} O \mathcal{F} W A\left(<d_{1}, d_{2}, \ldots, d_{n}>\right. \\
& \left.\left(\hbar_{1}, \hbar_{2}, \ldots, \hbar_{m}\right)\right)=\begin{array}{l}
q-\mathcal{R} O \mathcal{F} W A\left(\hbar_{1}, \hbar_{2}, \ldots, \hbar_{m}\right) \otimes \\
q-\mathcal{R} O \mathcal{F} W A\left(d_{1}, d_{2}, \ldots, d_{n}\right)
\end{array}
\end{aligned}
$$

The aggregation result for $\mathrm{q}-\mathcal{R} \mathrm{O} \mathcal{F} \mathrm{Ns}$ through operation rules is described as in Theorem 10 .

Theorem 10 Let $\hbar_{k}=\left(\mu_{\hbar_{k}}, \eta_{\hbar_{k}}\right)(k=1,2, \ldots, m)$ be the preferences suggested by the senior experts for the $q-\mathcal{R} O \mathcal{F} N s$ $d_{\ell}=\left(\mu_{d_{\ell}}, \eta_{d_{\ell}}\right)(\ell=1,2, \ldots, n)$, having weight vector $\hat{u}=$ $\left(\hat{u}_{1}, \hat{u}_{2}, \ldots, \hat{u}_{m}\right)^{\mathrm{T}}$ with $\sum_{k=1}^{m} \hat{u}_{k}=1$ where $\hat{u}_{k} \in[0,1]$. Let $u=\left(u_{1}, u_{2}, \ldots, u_{n}\right)^{\mathrm{T}}$ with $\sum_{\ell=1}^{n} u_{\ell}=1$ where $u_{\ell} \in$ $[0,1]$, be the associated weight vector for $q-\mathcal{R O F} N s d_{\ell}=$ $\left(\mu_{d_{\ell}}, \eta_{d_{\ell}}\right)$, then $G G q$-ROF $O \mathcal{F}$ operator is given as

$$
\begin{aligned}
& G G q-\mathcal{R} O \mathcal{F} W A\left(<d_{1}, d_{2}, \ldots, d_{n}>,\left(\hbar_{1}, \hbar_{2}, \ldots, \hbar_{m}\right)\right) \\
& =\left(\underset{k=1}{\oplus} \hat{u}_{k} \hbar_{k}\right) \otimes\left(\bigoplus_{\ell=1}^{n} u_{\ell} d_{\ell}\right) \text {. } \\
& =\left(\begin{array}{c}
\sqrt[q]{1-\prod_{k=1}^{m}\left(1-\mu_{\hbar_{k}}^{q}\right)^{\hat{u}_{k}}} \cdot \sqrt[q]{1-\prod_{\ell=1}^{n}\left(1-\mu_{d_{\ell}}^{q}\right)^{u_{\ell}}}, \\
\sqrt[q]{\prod_{k=1}^{m}\left(\eta_{\hbar_{k}}^{\hat{u}_{k}}\right)^{q}+\left(1-\prod_{k=1}^{m}\left(\eta_{\hbar_{k}}^{\hat{u}_{k}}\right)^{q}\right) \prod_{\ell=1}^{n}\left(\eta_{d_{\ell}}^{u_{\ell}}\right)^{q}}
\end{array}\right)
\end{aligned}
$$

Proof We use mathematical induction to prove this theorem. Now for $n=2$ we get

$$
\begin{aligned}
& \text { GGq- } \mathcal{R} O \mathcal{F} W A\left(<d_{1}, d_{2}>,\left(\hbar_{1}, \hbar_{2}, \ldots, \hbar_{m}\right)\right) \\
& =\left(\bigoplus_{k=1}^{m} \hat{u}_{k} \hbar_{k}\right) \otimes\left(u_{1} d_{1} \oplus u_{2} d_{2}\right) \\
& =\left(\sqrt[q]{1-\prod_{k=1}^{m}\left(1-\mu_{\hbar_{k}}^{q}\right)^{\hat{u}_{k}}}, \prod_{k=1}^{m} \eta_{\hbar_{k}}^{\hat{u}_{k}}\right) \otimes\left(u_{1} d_{1} \oplus u_{2} d_{2}\right) \\
& =\left(\sqrt[q]{1-\prod_{k=1}^{m}\left(1-\mu_{\hbar_{k}}^{q}\right)^{\hat{u}_{k}}}, \prod_{k=1}^{m} \eta_{\hbar_{k}}^{\hat{u}_{k}}\right) \\
& \otimes\left(\sqrt[q]{1-\prod_{\ell=1}^{2}\left(1-\mu_{d_{\ell}}^{q}\right)^{u_{\ell}}}, \prod_{\ell=1}^{2} \eta_{d_{\ell}}^{u_{\ell}}\right)
\end{aligned}
$$$$
=\left(\sqrt[q]{1-\prod_{k=1}^{m}\left(1-\mu_{\hbar_{k}}^{q}\right)^{\hat{u}_{k}}} \cdot \sqrt[q]{1-\prod_{\ell=1}^{2}\left(1-\mu_{d_{\ell}}^{q}\right)^{u_{\ell}}},\right.
$$

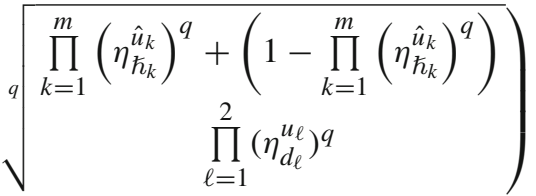

this implies for $n=2$ the result holds.

Next assume that the result holds for $n=K$, that is

$$
\begin{aligned}
& \text { GGq- } \mathcal{R} O \mathcal{F} W A\left(<d_{1}, d_{2}, \ldots, d_{K}>,\left(\hbar_{1}, \hbar_{2}, \ldots, \hbar_{m}\right)\right) \\
& =\left(\underset{k=1}{\oplus} \hat{u}_{k} \hbar_{k}\right) \otimes\left(\underset{\ell=1}{\bigoplus_{\ell}} u_{K} d_{K}\right)
\end{aligned}
$$

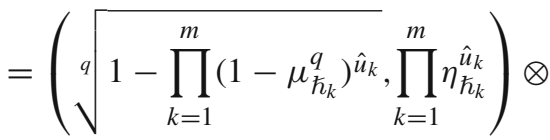

$$
\begin{aligned}
& \left(\sqrt[q]{1-\prod_{\ell=1}^{K}\left(1-\mu_{d_{\ell}}^{q}\right)^{u_{\ell}}, \prod_{\ell=1}^{K} \eta_{d_{\ell}}^{u_{\ell}}}\right) \\
& =\left(\sqrt[q]{1-\prod_{k=1}^{m}\left(1-\mu_{\hbar_{k}}^{q}\right)^{\hat{u}_{k}}} \cdot \sqrt[q]{1-\prod_{\ell=1}^{K}\left(1-\mu_{d_{\ell}}^{q}\right)^{u_{\ell}}}\right. \text {, } \\
& \sqrt{\frac{\prod_{k=1}^{m}\left(\eta_{\hbar_{k}}^{\hat{u}_{k}}\right)^{q}+\left(1-\prod_{k=1}^{m}\left(\eta_{\hbar_{k}}^{\hat{u}_{k}}\right)^{q}\right)}{\prod_{\ell=1}^{K}\left(\eta_{d_{\ell}}^{u_{\ell}}\right)^{q}}}
\end{aligned}
$$

Now to show that the result hold for $n=K+1$, then we have

GGq- $\mathcal{R} O \mathcal{F} W A\left(<d_{1}, d_{2}, \ldots, d_{K}, d_{K+1}>,\left(\hbar_{1}, \hbar_{2}, \ldots, \hbar_{m}\right)\right)$ $=\left(\underset{k=1}{\oplus} \hat{u}_{k} \hbar_{k}\right) \otimes\left(u_{1} d_{1} \oplus u_{2} d_{2} \oplus \cdots \oplus u_{K} d_{K} \oplus u_{K+1} d_{K+1}\right)$ $=\left(\underset{k=1}{\oplus} \hat{u}_{k} \hbar_{k}\right) \otimes\left\{\left(u_{1} d_{1} \oplus u_{2} d_{2} \oplus \cdots \oplus u_{K} d_{K}\right) \oplus u_{K+1} d_{K+1}\right\}$

$$
\begin{aligned}
& =\left(\sqrt[q]{1-\prod_{k=1}^{m}\left(1-\mu_{\hbar_{k}}^{q}\right)^{\hat{u}_{k}}, \prod_{k=1}^{m} \eta_{\hbar_{k}}^{\hat{u}_{k}}}\right) \\
& \otimes\left\{\begin{array}{c}
\left.\left(\sqrt[q]{1-\prod_{\ell=1}^{k}\left(1-\mu_{d_{\ell}}^{q}\right)^{u_{\ell}}, \prod_{\ell=1}^{k}\left(\eta_{d_{\ell}}^{u_{\ell}}\right)}\right) \oplus\right\} \\
\left(\sqrt[q]{1-\left(1-\mu_{d_{k+1}}^{q}\right)^{u_{k+1}}}, \eta_{d_{k+1}}^{u_{k+1}}\right)
\end{array}\right\} \\
& =\left(\begin{array}{c}
\left.\sqrt[q]{1-\prod_{k=1}^{m}\left(1-\mu_{\hbar_{k}}^{q}\right)^{\hat{u}_{k}}, \prod_{k=1}^{m} \eta_{\hbar_{k}}^{\hat{u}_{k}}}\right) \\
\sqrt[q]{1-\prod_{\ell=1}^{k}\left(1-\mu_{d_{\ell}}^{q}\right)^{u_{\ell}} \cdot\left(1-\mu_{d_{k+1}}^{q}\right)^{u_{k+1}},} \\
\prod_{\ell=1}^{k}\left(\eta_{d_{\ell}}^{u_{\ell}}\right) \cdot \eta_{d_{k+1}}^{u_{k+1}}
\end{array}\right)
\end{aligned}
$$




$$
\begin{aligned}
& =\left(\sqrt[q]{1-\prod_{k=1}^{m}\left(1-\mu_{\hbar_{k}}^{q}\right)^{\hat{u}_{k}}}, \prod_{k=1}^{m} \eta_{\hbar_{k}}^{\hat{u}_{k}}\right) \\
& \otimes\left(\sqrt[q]{\left.1-\prod_{\ell=1}^{K+1}\left(1-\mu_{d_{\ell}}^{q}\right)^{u_{\ell}}, \prod_{\ell=1}^{K+1} \eta_{d_{\ell}}^{u_{\ell}}\right)}\right. \\
& =\left(\sqrt[q]{1-\prod_{k=1}^{m}\left(1-\mu_{\hbar_{k}}^{q}\right)^{\hat{u}_{k}} \cdot \sqrt[q]{1-\prod_{\ell=1}^{K+1}\left(1-\mu_{d_{\ell}}^{q}\right)^{u_{\ell}},}}\right. \\
& \left.\sqrt[q]{\prod_{k=1}^{m}\left(\eta_{\hbar_{k}}^{\hat{u}_{k}}\right)^{q}+\left(1-\prod_{k=1}^{m}\left(\eta_{\hbar_{k}}^{\hat{u}_{k}}\right)^{q}\right)}\right) \text { this implies that } n \text { is } \\
& \prod_{\ell=1}^{K+1}\left(\eta_{d_{\ell}}^{u_{\ell}}\right)^{q}
\end{aligned}
$$

true for $K+1$. Therefore, the given result is holds for any number of q-ROFNs on the based of expert preferences.

Moreover, the aggregated result achieved from GGq$\mathcal{R O} \mathcal{F}$ WA is also a q- $\mathcal{R O} \mathcal{F}$ Ns.

Remark 5 (a) If the generalized parameter $p_{k}=(1,0)$ ( for all $k=1,2, \ldots, m)$, and $q=1$, so in this case the GGq$\mathcal{R O} \mathcal{F}$ WA operator degenerates to $\mathrm{I} \mathcal{F}$ WA operator.

(b) If the generalized parameter $p_{k}=(1,0)$ (for all $k=1,2, \ldots, m)$, and $q=2$, then the GGq- $\mathcal{R} O \mathcal{F}$ WA operator degenerates to $\mathrm{P} \mathcal{F} W A$ operator. (c) If the assign value $q=2$ is fixed then the GGq- $\mathcal{R} O \mathcal{F} W A$ operator reduces to GGP $\mathcal{F}$ WA operator.

Theorem 11 Let $\hbar_{k}=\left(\mu_{\hbar_{k}}, \eta_{\hbar_{k}}\right)(k=1,2, \ldots, m)$ be the preferences suggested by the senior experts for the $q-\mathcal{R} O \mathcal{F} N s$ $d_{\ell}=\left(\mu_{d_{\ell}}, \eta_{d_{\ell}}\right)(\ell=1,2, \ldots, n)$, having weight vector $\hat{u}=$ $\left(\hat{u}_{1}, \hat{u}_{2}, \ldots, \hat{u}_{m}\right)^{\mathrm{T}}$ with $\sum_{k=1}^{m} \hat{u}_{k}=1$ where $\hat{u}_{k} \in[0,1]$. Let $u=\left(u_{1}, u_{2}, \ldots, u_{n}\right)^{\mathrm{T}}$ with $\sum_{\ell=1}^{n} u_{\ell}=1$ where $u_{\ell} \in$ $[0,1]$, be the associated weight vector for $q-\mathcal{R} O \mathcal{F} N s d_{\ell}=$ $\left(\mu_{d_{\ell}}, \eta_{d_{\ell}}\right)$, then for $G G q-\mathcal{R} O \mathcal{F} W A$ operator the following assertions are hold:

i: (Idempotency): If $d_{\ell}=d$ (for all $\left.\ell=1,2,3, \ldots, n\right)$, and $\hbar_{k}=\hbar($ for all $k=1,2, \ldots, m)$ then

$$
\begin{aligned}
& G G q-\mathcal{R O F W} A\left(<d_{1}, d_{2}, d_{3}, \ldots, d_{n}>\right. \\
& \left.\left(\hbar_{1}, \hbar_{2}, \ldots, \hbar_{m}\right)\right)=\hbar \otimes d .
\end{aligned}
$$

ii: (Boundarycondition): If $d_{\ell}^{-}=\left(\min \mu_{\hbar_{k} \otimes d_{\ell}}, \max \eta_{\hbar_{k} \otimes d_{\ell}}\right)$ and $d_{\ell}^{+}=\left(\max \mu_{\hbar_{k} \otimes d_{\ell}}, \min \eta_{\hbar_{k} \otimes d_{\ell}}\right)(\forall \ell=1,2,3, \ldots, n)$ $(\forall k=1,2,3, \ldots, m)$, then

$$
\begin{aligned}
& d_{\ell}^{-} \leq G G q-\mathcal{R O F} \mathcal{F} A\left(<d_{1}, d_{2}, d_{3}, \ldots, d_{n}>\right. \\
& \left.\left(\hbar_{1}, \hbar_{2}, \ldots, \hbar_{m}\right)\right) \leq d_{\ell}^{+} .
\end{aligned}
$$

iii: (Monotonicity): Suppose $d_{\ell}=\left(\mu_{d_{\ell}}, \eta_{d_{\ell}}\right)$ and $d_{\ell}^{*}=$ $\left(\mu_{d_{\ell}^{*}}, \eta_{d_{\ell}^{*}}\right)(\ell=1,2, \ldots, n)$ be two collection of $n q-$ ROFNs such that $\mu_{d_{\ell}} \leq \mu_{d_{\ell}^{*}}$ and $\eta_{d_{\ell}} \geq \eta_{d_{\ell}^{*}}$, then $G G q-\mathcal{R} O \mathcal{F W} A\left(<d_{1}, d_{2}, d_{3}, \ldots, d_{n}>\right.$, $\left.\left(\hbar_{1}, \hbar_{2}, \ldots, \hbar_{m}\right)\right) \leq G G q-\mathcal{R} O \mathcal{F} \mathcal{W} A$ $\left(<d_{1}^{*}, d_{2}^{*}, d_{3}^{*}, \ldots, d_{n}^{*}>,\left(\hbar_{1}, \hbar_{2}, \ldots, \hbar_{m}\right)\right)$. iv: (Commutativity): Suppose $d_{\ell}=\left(\mu_{d_{\ell}}, \eta_{d_{\ell}}\right)$ and $\tilde{d}_{\ell}=$ $\left(\mu_{\tilde{d}_{\ell}}, \eta_{\tilde{d}_{\ell}}\right)(\ell=1,2, \ldots, n)$ be two collection of $n q-$ ROFNs where $\tilde{d}_{\ell}(\ell=1,2, \ldots, n)$ is the $\ell$ th largest object of $d_{\ell}$, then

$G G q-\mathcal{R} O \mathcal{F} \mathcal{W} A\left(<d_{1}, d_{2}, d_{3}, \ldots, d_{n}>\right.$,

$\left.\left(\hbar_{1}, \hbar_{2}, \ldots, \hbar_{m}\right)\right)=G G q-\mathcal{R} O \mathcal{F} \mathcal{W} A$

$\left(<\tilde{d}_{1}, \tilde{d}_{2}, \tilde{d}_{3}, \ldots, \tilde{d}_{n}>,\left(\hbar_{1}, \hbar_{2}, \ldots, \hbar_{m}\right)\right)$.

Proof Straightforward.

Proposition 4 (a) If the priority of the senior decision maker/expert about generalized parameter is taken as $\hbar_{k}=$ $(1,0)($ for all $k=1,2, \ldots, m)$, then the proposed $G G q$ $\mathcal{R} O \mathcal{F W}$ A operator reduces to $q-\mathcal{R} O \mathcal{F} \mathcal{W}$ A operator.

(b) If the priority of the senior decision maker/expert about generalized parameter is taken as $\hbar_{k}=(0,1)($ for all $k=$ $1,2, \ldots, m)$, then the proposed $G G q-\mathcal{R} O \mathcal{F} \mathcal{W} A$ operator presents the same result $(0,1)$.

Proof Proofs are straightforward.

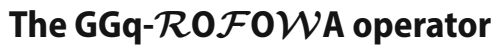

From GGq- $\mathcal{R O} \mathcal{F} W A$, it is clear that in GGq- $\mathcal{R} O \mathcal{F}$ WA operators just the $\mathrm{q}-\mathcal{R} \mathrm{O} \mathcal{F}$ values are weighed on the basis of group generalized parameter, while the GGq- $\mathcal{R} O \mathcal{F} O W A$ operator weights the ordered positions after scoring the q- $\mathcal{R O} \mathcal{F}$ values rather than weighting the $\mathrm{q}-\mathcal{R} \mathrm{O} \mathcal{F}$ values themselves on the base of group generalized parameter. Therefore, here, we will present the detail study of $\mathrm{GGq}-\mathcal{R O} \mathcal{F} O \mathcal{W A}$ operator and their properties.

Definition 13 Consider a group of experts/observers who justify the information under the $\mathrm{q}-\mathcal{R} O \mathcal{F}$ environment. Let $\hbar_{k}=\left(\mu_{\hbar_{k}}, \eta_{\hbar_{k}}\right)(k=1,2, \ldots, m)$ be the priorities/preferences suggested by the senior experts for the q- $\mathcal{R O} \mathcal{F}$ Ns $d_{\ell}=\left(\mu_{d_{\ell}}, \eta_{d_{\ell}}\right)(\ell=1,2, \ldots, n)$, then the GGq$\mathcal{R O} \mathcal{F O W A}$ operators is given as:

$$
\begin{aligned}
& \operatorname{GGq} \mathcal{R} O \mathcal{F} O \mathcal{W} A\left(<d_{1}, d_{2}, \ldots, d_{n}>,\left(\hbar_{1}, \hbar_{2}, \ldots, \hbar_{m}\right)\right) \\
& =\begin{array}{l}
q-\mathcal{R} O \mathcal{F} W A\left(\hbar_{1}, \hbar_{2}, \ldots, \hbar_{m}\right) \otimes \\
q-\mathcal{R} O \mathcal{F} O W A\left(d_{1}, d_{2}, \ldots, d_{n}\right)
\end{array}
\end{aligned}
$$

The aggregation result for $\mathrm{q}-\mathcal{R} \mathrm{O} \mathcal{F} \mathrm{Ns}$ through operation rules is described as in Theorem 12 .

Theorem 12 Suppose that $\hbar_{k}=\left(\mu_{\hbar_{k}}, \eta_{\hbar_{k}}\right)(k=1,2, \ldots, m)$ be the priorities/preferences suggested by the senior experts for the $q$-ROF $N s d_{\ell}=\left(\mu_{d_{\ell}}, \eta_{d_{\ell}}\right)(\ell=1,2, \ldots, n)$, having weight vector $\hat{u}=\left(\hat{u}_{1}, \hat{u}_{2}, \ldots, \hat{u}_{n}\right)^{\mathrm{T}}$ with $\sum_{k=1}^{m} \hat{u}_{k}=$ 1 where $\hat{u}_{k} \in[0,1]$. Let $u=\left(u_{1}, u_{2}, \ldots, u_{n}\right)^{\mathrm{T}}$ with $\sum_{\ell=1}^{n} u_{\ell}=1$ where $u_{\ell} \in[0,1]$, be the associated 
weight vector for $q$-R $\mathcal{R} O \mathcal{F} N s d_{\ell}=\left(\mu_{d_{\ell}}, \eta_{d_{\ell}}\right)$, then $G G q$ $\mathcal{R O F} O \mathcal{W} A$ operator is given as;

$$
\begin{aligned}
& G G q-\mathcal{R} O \mathcal{F} O \mathcal{W} A\left(<d_{1}, d_{2}, \ldots, d_{n}>,\left(\hbar_{1}, \hbar_{2}, \ldots, \hbar_{m}\right)\right) \\
& =\left(\underset{k=1}{\oplus} \hat{u}_{k} \hbar_{k}\right) \otimes\left(\bigoplus_{\ell=1}^{n} u_{\ell} \tilde{d}_{\ell}\right) . \\
& =\left(\frac{\sqrt[q]{1-\prod_{k=1}^{m}\left(1-\mu_{\hbar_{k}}^{q}\right)^{\hat{u}_{k}}} \cdot \sqrt[q]{1-\prod_{\ell=1}^{n}\left(1-\mu_{\tilde{d}_{\ell}}^{q}\right)^{u_{\ell}}},}{\sqrt[q]{\prod_{k=1}^{m}\left(\eta_{\hbar_{k}}^{\hat{u}_{k}}\right)^{q}+\left(1-\prod_{k=1}^{m}\left(\eta_{\hbar_{k}}^{\hat{u}_{k}}\right)^{q}\right) \prod_{\ell=1}^{n}\left(\eta_{\tilde{d}_{\ell}}^{u_{\ell}}\right)^{q}}}\right)
\end{aligned}
$$

where $\tilde{d}_{\ell}=\left(\mu_{\tilde{d}_{\ell}}, \eta_{\tilde{d}_{\ell}}\right)$ indicate the $\ell$ th largest object of $n$ $q$ - $\mathcal{R O F N s} d_{\ell}=\left(\mu_{d_{\ell}}, \eta_{d_{\ell}}\right)$.

Proof Proof is easy and directly follows form Theorem 10.

Remark 6 (a) If the generalized parameter $p_{k}=(1,0)$ ( for all $k=1,2, \ldots, m)$, and $q=1$, then the GGq- $\mathcal{R O} \mathcal{F} O \mathcal{W A}$ operator reduces to $\mathrm{I} \mathcal{F} \mathcal{W}$ A operator.

(b) If the generalized parameter $p_{k}=(1,0)$ (for all $k=1,2, \ldots, m)$, and $q=2$, then the GGq- $\mathcal{R O} \mathcal{F} O \mathcal{W A}$ operator reduces to $\mathrm{P} \mathcal{F} O \mathcal{W A}$ operator.

(c) If the value of parameter $q=2$ is fixed then the GGq$\mathcal{R O} \mathcal{F} O \mathcal{W}$ A operator reduces to GGP $\mathcal{F} O \mathcal{W A}$ operator.

Theorem 13 Let $\hbar_{k}=\left(\mu_{\hbar_{k}}, \eta_{\hbar_{k}}\right)(k=1,2, \ldots, m)$ be the priorities/preferences suggested by the senior experts for the $q$-ROF $N s d_{\ell}=\left(\mu_{d_{\ell}}, \eta_{d_{\ell}}\right)(\ell=1,2, \ldots, n)$, having weight vector $\hat{u}=\left(\hat{u}_{1}, \hat{u}_{2}, \ldots, \hat{u}_{n}\right)^{\mathrm{T}}$ with $\sum_{k=1}^{m} \hat{u}_{k}=1$ where $\hat{u}_{k} \in$ $[0,1]$. Let $u=\left(u_{1}, u_{2}, \ldots, u_{n}\right)^{\mathrm{T}}$ with $\sum_{\ell=1}^{n} u_{\ell}=1$ where $u_{\ell} \in[0,1]$, be the associated weight vector for $q-\mathcal{R} O \mathcal{F} N s$

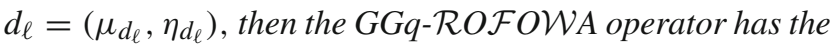
properties:

i: (Idempotency): If $\tilde{d}_{\ell}=\tilde{d}$ (for all $\left.\ell=1,2,3, \ldots, n\right)$, and $\hbar_{k}=\hbar$ (for all $\left.k=1,2, \ldots, m\right)$ then

$$
\begin{aligned}
& G G q-\mathcal{R} O \mathcal{F} O \mathcal{W} A\left(<d_{1}, d_{2}, d_{3}, \ldots, d_{n}>\right. \\
& \left.\quad\left(\hbar_{1}, \hbar_{2}, \ldots, \hbar_{m}\right)\right) \\
& =\hbar \otimes \tilde{d}
\end{aligned}
$$

ii: (Boundary condition): If $\tilde{d}_{\ell}^{-}=$

$$
\begin{aligned}
& \left(\min \mu_{\hbar_{k} \otimes \tilde{d}_{\ell}}, \max \eta_{\hbar_{k} \otimes \tilde{d}_{\ell}}\right) \text { and } \\
& \tilde{d}_{\ell}^{+}=\left(\max \mu_{\hbar_{k} \otimes \tilde{d}_{\ell}}, \min \eta_{\hbar_{k} \otimes \tilde{d}_{\ell}}\right) \\
& (\forall \ell=1,2,3, \ldots, n) \\
& (\forall k=1,2,3, \ldots, m), \text { then } \\
& \tilde{d}_{\ell}^{-} \leq G G q-\mathcal{R} O \mathcal{F} O \mathcal{W} A\left(<d_{1}, d_{2}, d_{3}, \ldots, d_{n}>,\right. \\
& \left.\quad\left(\hbar_{1}, \hbar_{2}, \ldots, \hbar_{m}\right)\right) \leq \tilde{d}_{\ell}^{+} .
\end{aligned}
$$

iii: (Monotonicity): Suppose $d_{\ell}=\left(\mu_{d_{\ell}}, \eta_{d_{\ell}}\right)$ and $d_{\ell}^{*}=$ $\left(\mu_{d_{\ell}^{*}}, \eta_{d_{\ell}^{*}}\right)(\ell=1,2, \ldots, n)$ be two collection of $n q-$ ROFNs such that $\mu_{d_{\ell}} \leq \mu_{d_{\ell}^{*}}$ and $\eta_{d_{\ell}} \geq \eta_{d_{\ell}^{*}}$, then

$G G q-\mathcal{R} O \mathcal{F} O \mathcal{W} A\left(<d_{1}, d_{2}, d_{3}, \ldots, d_{n}>\right.$,

$\left.\left(\hbar_{1}, \hbar_{2}, \ldots, \hbar_{m}\right)\right) \leq G G q-\mathcal{R} O \mathcal{F} O \mathcal{W} A$

$\left(<d_{1}^{*}, d_{2}^{*}, d_{3}^{*}, \ldots, d_{n}^{*}>,\left(\hbar_{1}, \hbar_{2}, \ldots, \hbar_{m}\right)\right)$.

iv: (Commutativity): Suppose $d_{\ell}=\left(\mu_{d_{\ell}}, \eta_{d_{\ell}}\right)$ and $\tilde{d}_{\ell}=$ $\left(\mu_{\tilde{d}_{\ell}}, \eta_{\tilde{d}_{\ell}}\right)(\ell=1,2, \ldots, n)$ be two collection of $n q-$ ROFNs where $\tilde{d}_{\ell}(\ell=1,2, \ldots, n)$ is represents the $\ell t h$ largest object of $d_{\ell}$, then

$G G q-\mathcal{R} O \mathcal{F} O \mathcal{W} A\left(<d_{1}, d_{2}, d_{3}, \ldots, d_{n}>\right.$,

$\left.\left(\hbar_{1}, \hbar_{2}, \ldots, \hbar_{m}\right)\right)=G G q-\mathcal{R} O \mathcal{F} O \mathcal{W} A$

$\left(<\tilde{d}_{1}, \tilde{d}_{2}, \tilde{d}_{3}, \ldots, \tilde{d}_{n}>,\left(\hbar_{1}, \hbar_{2}, \ldots, \hbar_{m}\right)\right)$.

Proof Proofs is straightforward.

Proposition 5 (a) If the priorities/preferences of the senior decision makers/experts about generalized parameters are taken as $\hbar_{k}=(1,0)(k=1,2, \ldots, m)$, then the proposed GGq-ROF $O \mathcal{W} A$ operator reduces to $q$-ROF $O \mathcal{W} A$ operator.

(b) If the priorities/preferences of the senior decision makers/experts about generalized parameters are taken as $\hbar_{k}=(0,1)(k=1,2, \ldots, m)$, then the proposed $G G q$ $\mathcal{R} O \mathcal{F} O \mathcal{W} A$ operator presents the same result $(0,1)$.

Proof Straightforward.

\section{The GGq- $\mathcal{R} 0 \mathcal{F}$ HA operator}

From the above discussion, it is concluded that the GGq$\mathcal{R O} \mathcal{F} \mathcal{W}$ A operator just weights the $\mathrm{q}-\mathcal{R O} \mathcal{F}$ values on the basis of group generalized parameter, and similarly the GGq$\mathcal{R O} \mathcal{F} O \mathcal{W}$ A operator just weights the ordered positions after scoring the q- $\mathcal{R O} \mathcal{F}$ values rather than weighing the $\mathrm{q}-\mathcal{R} \mathrm{O} \mathcal{F}$ values themselves on the basis of group generalized parameter. Therefore, it is clear that the weights denote distinct attributes in both $\mathrm{GGq}-\mathcal{R} O \mathcal{F} \mathcal{W}$ A and $\mathrm{GGq}-\mathcal{R O} \mathcal{F} O \mathcal{W A}$ operators. However, at the same time both the operators weighs just one of them. To handle this restriction, here we will originate the study of GGq- $\mathcal{R O} \mathcal{F} H A$ operator, which weighs both the given values at the same time, that is q$\mathcal{R O} \mathcal{F}$ values and its ordered position on the basis of group generalized parameter.

Definition 14 Consider a group of experts/observers who justify the information under the $\mathrm{q}-\mathcal{R} \mathrm{O} \mathcal{F}$ environment. Let $\hbar_{k}=\left(\mu_{\hbar_{k}}, \eta_{\hbar_{k}}\right)(k=1,2, \ldots, m)$ be the priorities/preferences suggested by the senior experts for the $\mathrm{q}-\mathcal{R O} \mathcal{F}$ Ns $d_{\ell}=\left(\mu_{d_{\ell}}, \eta_{d_{\ell}}\right)(\ell=1,2, \ldots, n)$, then the GGq$\mathcal{R O} \mathcal{F H A}$ operator is given as:

$\operatorname{GGq}-\mathcal{R} O \mathcal{F} H A\left(<d_{1}, d_{2}, \ldots, d_{n}>,\left(\hbar_{1}, \hbar_{2}, \ldots, \hbar_{m}\right)\right)$ 


$$
=\begin{aligned}
& q-\mathcal{R} O \mathcal{F} H A\left(\hbar_{1}, \hbar_{2}, \ldots, \hbar_{m}\right) \otimes \\
& q-\mathcal{R} O \mathcal{F} H A\left(d_{1}, d_{2}, \ldots, d_{n}\right)
\end{aligned}
$$

The aggregation result for $\mathrm{q}-\mathcal{R} \mathrm{O} \mathcal{F} \mathrm{Ns}$ through operation rules is described as in Theorem 14.

Theorem 14 Let $\hbar_{k}=\left(\mu_{\hbar_{k}}, \eta \hbar_{k}\right)(k=1,2, \ldots, m)$ be the priorities/preferences suggested by the senior experts for the $q$ - $\mathcal{R} O \mathcal{F} N s d_{\ell}=\left(\mu_{d_{\ell}}, \eta_{d_{\ell}}\right)(\ell=1,2, \ldots, n)$, having weight vector $\hat{u}=\left(\hat{u}_{1}, \hat{u}_{2}, \ldots, \hat{u}_{n}\right)^{\mathrm{T}}$ with $\sum_{k=1}^{m} \hat{u}_{k}=1$ where $\hat{u}_{k} \in$ $[0,1]$. Let $\ddot{u}=\left(\ddot{u}_{1}, \tilde{u}_{2}, \ldots, \tilde{u}_{n}\right)^{\mathrm{T}}$ with $\sum_{\ell=1}^{n} \ddot{u}_{\ell}=1$ where $\tilde{u}_{\ell} \in[0,1]$, be the weight vector and $u=\left(u_{1}, u_{2}, \ldots, u_{n}\right)^{\mathrm{T}}$ with $\sum_{\ell=1}^{n} u_{\ell}=1$ where $u_{\ell} \in[0,1]$, be the associated weight vector of $q$-ROF $O \mathcal{F} d_{\ell}=\left(\mu_{d_{\ell}}, \eta_{d_{\ell}}\right)$, then GGq$\mathcal{R} O \mathcal{F} H A$ operator is given as;

$$
\begin{aligned}
& G G q-\mathcal{R} O \mathcal{F} H A\left(<d_{1}, d_{2}, \ldots, d_{n}>,\left(\hbar_{1}, \hbar_{2}, \ldots, \hbar_{m}\right)\right)
\end{aligned}
$$

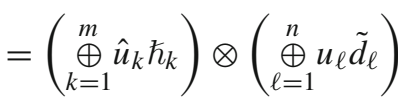

$$
\begin{aligned}
& =\left(\begin{array}{l}
\sqrt[q]{1-\prod_{k=1}^{m}\left(1-\mu_{\hbar_{k}}^{q}\right)^{\hat{u}_{k}}} \cdot \sqrt[q]{1-\prod_{\ell=1}^{n}\left(1-\mu_{\tilde{d}_{\ell}}^{q}\right)^{u_{\ell}}}, \\
\sqrt[q]{\prod_{k=1}^{m}\left(\eta_{\hbar_{k}}^{\hat{u}_{k}}\right)^{q}+\left(1-\prod_{k=1}^{m}\left(\eta_{\hbar_{k}}^{\hat{u}_{k}}\right)^{q}\right) \prod_{\ell=1}^{n}\left(\eta_{\tilde{d}_{\ell}}^{u_{\ell}}\right)^{q}}
\end{array}\right)
\end{aligned}
$$

where $\tilde{d}_{\ell}\left(\tilde{d}_{\ell}=n \tilde{u}_{\ell} d_{\ell}\right.$, for $\left.\ell=1,2, \ldots, n\right)$ indicate the permutation which is the lth largest object of the collection of $q$ - $\mathcal{R} O \mathcal{F} N s d_{\ell}=\left(\mu_{d_{\ell}}, \eta_{d_{\ell}}\right)(\ell=1,2, \ldots, n)$ and $n$ indicate the balancing coefficient.

Remark 7 (a) If the generalized parameter $p_{k}=(1,0)$ ( for all $k=1,2, \ldots, m)$, and $q=1$, then the $\mathrm{GGq}-\mathcal{R O} \mathcal{F} H \mathrm{~A}$ operator reduces to $\mathrm{I} \mathcal{F} H \mathrm{~A}$ operator.

(b) If the generalized parameter $p_{k}=(1,0)$ (for all $k=$ $1,2, \ldots, m)$, and $q=2$, then the GGq- $\mathcal{R O} \mathcal{F} H$ A operator reduces to $\mathrm{P} \mathcal{F} H \mathrm{~A}$ operator.

(c) If the value of parameter $q=2$ is fixed then the GGq$\mathcal{R O} \mathcal{F} H$ A operator reduces to GGP $\mathcal{F} H$ A operator.

Theorem 15 Let $\hbar_{k}=\left(\mu_{\hbar_{k}}, \eta_{\hbar_{k}}\right)(k=1,2, \ldots, m)$ be the priorities/preferences suggested by the senior experts for the $q$-ROF $N s d_{\ell}=\left(\mu_{d_{\ell}}, \eta_{d_{\ell}}\right)(\ell=1,2, \ldots, n)$, having weight vector $\hat{u}=\left(\hat{u}_{1}, \hat{u}_{2}, \ldots, \hat{u}_{n}\right)^{\mathrm{T}}$ with $\sum_{k=1}^{m} \hat{u}_{k}=1$ where $\hat{u}_{k} \in$ $[0,1]$. Let $\ddot{u}=\left(\ddot{u}_{1}, \tilde{u}_{2}, \ldots, \ddot{u}_{n}\right)^{\mathrm{T}}$ with $\sum_{\ell=1}^{n} \tilde{u}_{\ell}=1$ where $\tilde{u}_{\ell} \in[0,1]$, be the weight vector and $u=\left(u_{1}, u_{2}, \ldots, u_{n}\right)^{\mathrm{T}}$ with $\sum_{\ell=1}^{n} u_{\ell}=1$ where $u_{\ell} \in[0,1]$, be the associated weight vector of $q-\mathcal{R} O \mathcal{F} N s d_{\ell}=\left(\mu_{d_{\ell}}, \eta_{d_{\ell}}\right)$, then for $G G q$ $\mathcal{R} O \mathcal{F} H A$ operator the following conditions are hold:

i: (Idempotency): If $d_{\ell}=\tilde{d}$ (for all $\left.\ell=1,2,3, \ldots, n\right)$, and $\hbar_{k}=\hbar($ for all $k=1,2, \ldots, m)$ then

$$
\begin{aligned}
& G G q-\mathcal{R} O \mathcal{F} H A\left(<d_{1}, d_{2}, d_{3}, \ldots, d_{n}>,\right. \\
& \left.\left(\hbar_{1}, \hbar_{2}, \ldots, \hbar_{m}\right)\right)=\hbar \otimes \tilde{d} .
\end{aligned}
$$

ii: (Boundary condition): If $\tilde{d}_{\ell}^{-}=$

$$
\begin{aligned}
& \left(\min \mu_{\hbar_{k} \otimes \tilde{d}_{\ell}}, \max \eta_{\hbar_{k} \otimes \tilde{d}_{\ell}}\right) \text { and } \\
& \tilde{d}_{\ell}^{+}=\left(\max \mu_{\hbar_{k} \otimes \tilde{d}_{\ell}}, \min \eta_{\hbar_{k} \otimes \tilde{d}_{\ell}}\right) \\
& (\forall \ell=1,2,3, \ldots, n) \\
& (\forall k=1,2,3, \ldots, m), \text { then } \\
& \tilde{d}_{\ell}^{-} \leq G G q-\mathcal{R} O \mathcal{F} H A\left(<d_{1}, d_{2}, d_{3}, \ldots, d_{n}>,\right. \\
& \left.\quad\left(\hbar_{1}, \hbar_{2}, \ldots, \hbar_{m}\right)\right) \leq \tilde{d}_{\ell}^{+} .
\end{aligned}
$$

iii: (Monotonicity): Suppose $d_{\ell}=\left(\mu_{d_{\ell}}\right.$, $\left.\eta_{d_{\ell}}\right)$ and $d_{\ell}^{*}=\left(\mu_{d_{\ell}^{*}}, \eta_{d_{\ell}^{*}}\right)(\ell=1,2, \ldots, n)$ be two collection of $n q$-ROFNs such that $\mu_{d_{\ell}} \leq \mu_{d_{\ell}^{*}}$ and $\eta_{d_{\ell}} \geq$ $\eta_{d_{\ell}^{*}}$, then

$G G q-\mathcal{R} O \mathcal{F} H A\left(<d_{1}, d_{2}, d_{3}, \ldots, d_{n}>\right.$, $\left.\left(\hbar_{1}, \hbar_{2}, \ldots, \hbar_{m}\right)\right) \leq G G q-\mathcal{R} O \mathcal{F} H A\left(<d_{1}^{*}, d_{2}^{*}\right.$, $\left.d_{3}^{*}, \ldots, d_{n}^{*}>,\left(\hbar_{1}, \hbar_{2}, \ldots, \hbar_{m}\right)\right)$.

iv: (Commutativity): Suppose $d_{\ell}=\left(\mu_{d_{\ell}}, \eta_{d_{\ell}}\right)$ and $\tilde{d}_{\ell}=$ $\left(\mu_{\tilde{d}_{\ell}}, \eta_{\tilde{d}_{\ell}}\right)(\ell=1,2, \ldots, n)$ be two collection of $n q-$ ROFNs where $\tilde{d}_{\ell}(\ell=1,2, \ldots, n)$ is any permutation of $d_{\ell}$, then

$G G q-\mathcal{R} O \mathcal{F} H A\left(<d_{1}, d_{2}, d_{3}, \ldots, d_{n}>,\left(\hbar_{1}, \hbar_{2}\right.\right.$, $\left.\left.\ldots, \hbar_{m}\right)\right)=G G q-\mathcal{R O F} H A\left(<\tilde{d}_{1}, \tilde{d}_{2}, \tilde{d}_{3}, \ldots, \tilde{d}_{n}>\right.$ , $\left.\left(\hbar_{1}, \hbar_{2}, \ldots, \hbar_{m}\right)\right)$.

Proof Proofs are straightforward.

Proposition 6 (a) If the priority of the senior decision maker/expert about generalized parameter is taken as $\hbar=$ $(1,0)$, so in this case the proposed $G G q-\mathcal{R} O \mathcal{F} H$ A operator degenerates to $q-\mathcal{R} O \mathcal{F} H$ A operator.

(b) If the priority of the senior decision maker/expert about generalized parameter is taken as $\hbar=(0,1)$, then the proposed $G G q-\mathcal{R} O \mathcal{F} H A$ operator gives the result $(0,1)$.

Remark 8 (a) If $\tilde{u}=\left(\frac{1}{n}, \frac{1}{n}, \ldots, \frac{1}{n}\right)^{\mathrm{T}}$, so in this case the improved $G G q$-R $O \mathcal{F} H A$ operator degenerates to $G G q$ $\mathcal{R} O \mathcal{F} W A$ operator.

(b) If $u=\left(\frac{1}{n}, \frac{1}{n}, \ldots, \frac{1}{n}\right)^{\mathrm{T}}$, so in this case the proposed $G G q$ - $\mathcal{R} O \mathcal{F} H A$ operator degenerates to $G G q$ - $\mathcal{R} O \mathcal{F} O W A$ operator.

\section{An approach to MCDM and its application based on the generalization parameter}

In this section, the technique of MCDM is constructed on the concept of $\mathrm{GGq}-\mathcal{R O} \mathcal{F}$ information under generalized parameter. The general concept and steps of construction of the developed approach are given below. 


\section{MCDM approach}

To demonstrate the MCDM approach on the basis of developed approach, let $\mathcal{X}=\left\{d_{1}, d_{2}, \ldots, d_{m}\right\}$ be a certain discrete set of alternatives and $C=\left\{c_{1}, c_{2}, \ldots, c_{n}\right\}$ be the corresponding set of criteria having weight vector $u=$ $\left\{u_{1}, u_{2}, \ldots, u_{n}\right\}$ where $u_{i} \in[0,1]$ with $\sum_{i=1}^{n} u_{i}=1$, on the base of generalized parameter. A team of experts is called to for the judgement of each alternative $d_{i}(i=1,2, \ldots, m)$ to their corresponding criteria $c_{j}(j=1,2, \ldots, n)$. The experts give their assessment details in the form of q-R $\mathcal{R} \mathcal{F} \mathrm{Ns}$ denoted by $d_{i j}=\left(\mu_{d_{i j}}, \eta_{d_{i j}}\right)$ where in points of view of experts $\mu_{d_{i j}}$ represents the membership and, $\eta_{d_{i j}}$ represents the non-membership grades to which alternative $d_{i j}$ satisfies the the criteria $c_{j}$ having the condition that $0 \leq$ $\left(\mu_{d_{i j}}\right)^{q}+\left(\eta_{d_{i j}}\right)^{q} \leq 1$ for $q \geq 1$.

To certify the collected information in more accurate manner, the group $\left\{\hbar_{1}, \hbar_{2}, \ldots, \hbar_{k}\right\}$ of other senior experts are constituted which provide their preferences/ priorities for each alternative in the form of $\mathrm{q}-\mathcal{R} \mathrm{O} \mathcal{F} \mathrm{Ns}$ represented by $\hbar_{l}=\left(\mu \hbar_{l}, \eta_{\hbar_{l}}\right)(l=1,2, \ldots, k)$, with weight vector $\hat{u}=$ $\left(\hat{u}_{1}, \hat{u}_{2}, \ldots, \hat{u}_{k}\right)^{\mathrm{T}}$ such that $\hat{u}_{l} \in[0,1]$ with $\sum_{l=1}^{k} \hat{u}_{l}=1$.

\section{Algorithm}

The algorithm for the developed operator consists of the following steps.

step 1 From the above analysis collect the decision makers/expert's information provided for each alternative to their corresponding criteria and then construct a decision matrix

$$
[\mathcal{X}]_{m \times n}=\left(\begin{array}{cccc}
d_{11} & d_{12} & \cdots & d_{1 n} \\
d_{21} & d_{22} & \cdots & d_{2 n} \\
\vdots & \vdots & \ddots & \vdots \\
d_{m 1} & d_{m 2} & \cdots & d_{m n}
\end{array}\right),
$$

step 2 Collect the priorities/preferences of the senior's group experts against each alternatives under the generalized parameter and from these information construct the generalized parameter matrix

$$
[\mathcal{Y}]_{m \times k}=\left(\begin{array}{cccc}
\hbar_{11} & \hbar_{12} & \cdots & \hbar_{1 k} \\
\hbar_{21} & \hbar_{22} & \cdots & \hbar_{2 k} \\
\vdots & \vdots & \ddots & \vdots \\
\hbar_{m 1} & \hbar_{m 2} & \cdots & \hbar_{m k}
\end{array}\right),
$$

step 3 Append the matrices obtained from steps 1: and 2: to get the new matrix $[\mathcal{Z}]_{m \times(n+k)}$ which shows the priorities/preferences of expert's for each alternatives $d_{i j}$ corresponding to criteria $c_{j}$ on the bases of generalized parameter $\hbar_{l}$.

$$
[\mathcal{Z}]_{m \times(n+k)}=\left(\begin{array}{cccc}
d_{11} & d_{12} & \cdots & d_{1(n+k)} \\
d_{21} & d_{22} & \cdots & d_{2(n+k)} \\
\vdots & \vdots & \ddots & \vdots \\
d_{m 1} & d_{m 2} & \cdots & d_{m(n+k)}
\end{array}\right),
$$

step 4 Utilized the developed GGq- $\mathcal{R} O \mathcal{F}$ WA operator to get the overall aggregated result from matrix $[\mathcal{Z}]_{m \times(n+k)}$ for alternative $d_{i}$,

step 5 Determine the score of each aggregated result in step 4: and rank the results in a specific order to get the best alternative.

\section{Numerical illustration}

This section is devoted for the presentation of an illustrating example to demonstrate the validity and effectiveness of the developed approach with $\mathrm{q}-\mathcal{R} \mathrm{O} \mathcal{F}$ information.

Consider Pneumonia is a common disease and initially having four basic symptoms such as chest pain, fever, cough and fatigue. The disease of Pneumonia can be treated with four medicines. These medicines have various therapeutic effects on these four symptoms. Now for treating this disease, a doctor advise needs to analyze the best and worst therapeutic effects of these four medicines. Let $\mathcal{X}=\left\{d_{1}, d_{2}, d_{3}, d_{4}\right\}$ denotes the four medicines (alternatives) and $\mathcal{C}=\left\{c_{1}, c_{2}, c_{3}, c_{4}\right\}$ represents the fours symptoms (criteria). Furthermore, considering the significance degree of the four symptoms, the doctor provide the weight vector $u=\left(u_{1}=0.3, u_{2}=0.25, u_{3}=0.18, u_{4}=0.27\right)^{\mathrm{T}}$ of the criteria set. The doctor presents their evaluation for each medicine (alternative) to their corresponding symptom (criteria) in the form of $\mathrm{q}-\mathcal{R} \mathrm{O} \mathcal{F} \mathrm{Ns}$ which is given in Table 1. To justify the collected information in more accurate manner, consider a group of senior doctors/experts $\left\{\hbar_{1}, \hbar_{2}, \hbar_{3}\right\}$ provide their priority/preferences with weight vector $\hat{u}=$ $\left(\hat{u}_{1}=0.35, \hat{u}_{2}=0.32, \hat{u}_{3}=0.33\right)^{\mathrm{T}}$ such that $\hat{u}_{i} \in[0,1]$ with $\sum_{i=1}^{3} \hat{u}_{i}=1$. A group of senior doctors/experts provide their preferences report in the form of $\mathrm{q}-\mathcal{R O} \mathcal{F}$ Ns which is given in Table 2. The steps-wise algorithm of the presented approach for MCDM is given as

step 1 The collected information of decision maker/expert evaluation $[\mathcal{X}]_{m \times n}=\left(d_{i j}\right)_{m \times n}=\left(\mu_{d_{i j}}, \eta_{d_{i j}}\right)_{m \times n}$ for each alternative to their corresponding criteria are given in Table 1.

step 2 The preferences/priorities of the group of other senior decision makers/experts evaluation against each alternatives under the generalized parameter 
Table 1 q- $\mathcal{R O} \mathcal{F}$ expert's evaluation matrix $[\mathcal{X}]_{m \times n}$

\begin{tabular}{lllll}
\hline & $c_{1}$ & $c_{2}$ & $c_{3}$ & $c_{4}$ \\
\hline$d_{1}$ & $(0.9,0.2)$ & $(0.8,0.3)$ & $(0.95,0.15)$ & $(0.4,0.2)$ \\
$d_{2}$ & $(0.8,0.5)$ & $(0.7,0.2)$ & $(0.93,0.35)$ & $(0.6,0.1)$ \\
$d_{3}$ & $(0.9,0.3)$ & $(0.6,0.8)$ & $(0.85,0.52)$ & $(0.7,0.5)$ \\
$d_{4}$ & $(0.7,0.2)$ & $(0.9,0.4)$ & $(0.78,0.62)$ & $(0.5,0.3)$ \\
\hline
\end{tabular}

Table 2 Other senior expert's evaluation matrix $[\mathcal{Y}]_{m \times k}$ based on q$\mathcal{R O} \mathcal{F}$ gen- eralized Parameter

\begin{tabular}{llll}
\hline & $\hbar_{1}$ & $\hbar_{2}$ & $\hbar_{3}$ \\
\hline$d_{1}$ & $(0.91,0.23)$ & $(0.88,0.38)$ & $(0.9,0.2)$ \\
$d_{2}$ & $(0.85,0.5)$ & $(0.86,0.35)$ & $(0.7,0.3)$ \\
$d_{3}$ & $(0.9,0.3)$ & $(0.65,0.58)$ & $(0.6,0.4)$ \\
$d_{4}$ & $(0.76,0.25)$ & $(0.91,0.42)$ & $(0.5,0.6)$ \\
\hline
\end{tabular}

matrix $[\mathcal{Y}]_{m \times k}=\left[\hbar_{i l}\right]_{m \times k}=\left(\mu \hbar_{i l}, \eta \hbar_{i l}\right)_{m \times k}$ is given in Table 2.

step 3 Combine the matrices of steps 1: and 2: to get the new matrix $[\mathcal{Z}]_{m \times(n+k)}$ which shows the priorities/preferences of experts for each alternatives $d_{i j}$ corresponding to criteria $c_{j}$ on the bases of generalized parameter $\hbar_{l}$.

step 4 Utilized the developed GGq- $\mathcal{R O} \mathcal{F}$ WA operator to get the over all aggregated result from matrix $[\mathcal{Z}]_{m \times(n+k)}$ row wise for each alternative $d_{i}$.

Now the aggregated result for $d_{1}$ is as, for $q=3$;

$\xi_{1}=G G q-\mathcal{R} O \mathcal{F} W A\left(<d_{1}, d_{2}, \ldots, d_{n}>\right.$,

$\left.\left(\hbar_{1}, \hbar_{2}, \ldots, \hbar_{m}\right)\right)$

$\xi_{1}=\left(\begin{array}{l}\sqrt[q]{1-\prod_{k=1}^{m}\left(1-\mu_{\hbar_{k}}^{q}\right)^{\hat{u}_{k}} \cdot \sqrt[q]{1-\prod_{\ell=1}^{n}\left(1-\mu_{\mu_{d_{\ell}}}^{q}\right)^{u_{\ell}}},} \\ \sqrt[q]{\prod_{k=1}^{m}\left(\eta_{\eta \hbar_{k}}^{\hat{u}_{k}}\right)^{q}+\left(1-\prod_{k=1}^{m}\left(\eta_{\eta_{\hbar_{k}}}^{\hat{u}_{k}}\right)^{q}\right)_{\prod_{\ell=1}}^{n}\left(\eta_{d_{\ell}}^{u_{\ell}}\right)^{q}}\end{array}\right)$

$=(0.89792 \times 0.8477$,

$\sqrt[3]{\left.1.7156 \times 10^{-2}+0.98284 \times 9.2831 \times 10^{-3}\right)}$

$\xi_{1}=(0.76117,0.29731)$

Similarly we can find the others;

$\xi_{2}=(0.64677,0.40586), \xi_{3}=(0.62707,0.55885), \xi_{4}=$ $(0.61011,0.45385)$.

step 5 Determine the score of each aggregated result in step 4: and rank the results in descending order to get the best alternative.

$S\left(\xi_{1}\right)=0.41473, S\left(\xi_{2}\right)=0.20370, S\left(\xi_{3}\right)=$ $0.072038, S\left(\xi_{4}\right)=0.13362$

Hence from the score values we get the ranking result as; $d_{1} \geq d_{2} \geq d_{4} \geq d_{3}$. Therefore, from overall calculation it is clear that, the best medicine (alternative) against the given symptom (criteria) is $d_{1}$.

\section{Comparative analysis}

From the above analysis, it is clear that the best alternative to the corresponding criteria is $d_{1}$. If a single senior expert is recommended rather than a group of senior experts, which provide his preference/priority for the mention information, then the following results are concluded,

1: If the expert $\hbar_{1}$ is recommended for the consideration of mentioned information, then the score results are given as,

$$
\begin{aligned}
& S\left(\xi_{1}\right)=0.43771, S\left(\xi_{2}\right)=0.16556, S\left(\xi_{3}\right)=0.24166 \\
& S\left(\xi_{4}\right)=0.14959
\end{aligned}
$$

This implies that $d_{1} \geq d_{3} \geq d_{2} \geq d_{4}$.

2: If the expert $\hbar_{2}$ is recommended for the consideration of mentioned information, then the score results are given as,

$$
\begin{aligned}
& S\left(\xi_{1}\right)=0.35148, S\left(\xi_{2}\right)=0.25734, S\left(\xi_{3}\right)=-0.14418, \\
& S\left(\xi_{4}\right)=0.23582 .
\end{aligned}
$$

This implies that $d_{1} \geq d_{2} \geq d_{4} \geq d_{3}$.

3 : Similarly if the expert $\hbar_{3}$ is recommended for the consideration of mentioned information, then the score results are given as,

$$
\begin{aligned}
& S\left(\xi_{1}\right)=0.42686, S\left(\xi_{2}\right)=0.12847, S\left(\xi_{3}\right)=-0.058680 \\
& S\left(\xi_{4}\right)=-0.18629
\end{aligned}
$$

This implies that $d_{1} \geq d_{2} \geq d_{3} \geq d_{4}$.

The ranking results for the single expert for the same alternatives to their corresponding criteria is different but the best alternative remain same, which represents the importance of expert preferences, knowledge, consciousness and expertise on their preference values.

Moreover, by comparing the superiorities and advantages of the developed approach with existing methods in the literature using the same example and ignoring the group generalized parameter matrix $[\mathcal{Y}]_{m \times k}$. These methods including intuitionistic fuzzy weighted averaging (IFWA) operator presented by Xu [28] and Li [18], Pyhtagorean fuzzy weighted averaging (PFWA) operator presented by Yagger [32], Ma and $\mathrm{Xu}$ [22], symmetric Pythagorean fuzzy weighted averaging (SPFWA) operator presented by Ma and Xu [22], group generalized parameter Pythagorean fuzzy weighted averaging (GGPFWA) operator initiated by Joshi [17], q-ROFWA 
Table $3 \mathrm{GGq}-\mathcal{R} \mathrm{O} \mathcal{F}$ expert's priority/preferences matrix $[\mathcal{Z}]_{m \times(n+k)}$

Table 4 Comparative analysis of distinct aggregation operators

Table 5 Comparative analysis of distinct aggregation operators on $[\mathcal{Z}]_{m \times(n+k)}$

\begin{tabular}{llllllll}
\hline & $c_{1}$ & $c_{2}$ & $c_{3}$ & $c_{4}$ & $\hbar_{1}$ & $\hbar_{2}$ & $\hbar_{3}$ \\
\hline$d_{1}$ & $(0.9,0.2)$ & $(0.8,0.3)$ & $(0.95,0.15)$ & $(0.4,0.2)$ & $(0.91,0.23)$ & $(0.88,0.38)$ & $(0.9,0.2)$ \\
$d_{2}$ & $(0.8,0.5)$ & $(0.7,0.2)$ & $(0.93,0.35)$ & $(0.6,0.1)$ & $(0.85,0.5)$ & $(0.86,0.35)$ & $(0.7,0.3)$ \\
$d_{3}$ & $(0.9,0.3)$ & $(0.6,0.8)$ & $(0.85,0.52)$ & $(0.7,0.5)$ & $(0.9,0.3)$ & $(0.65,0.58)$ & $(0.6,0.4)$ \\
$d_{4}$ & $(0.7,0.2)$ & $(0.9,0.4)$ & $(0.78,0.62)$ & $(0.5,0.3)$ & $(0.76,0.25)$ & $(0.91,0.42)$ & $(0.5,0.6)$ \\
\hline
\end{tabular}

\begin{tabular}{llllll}
\hline Operators & \multicolumn{2}{l}{ Score values } & \multicolumn{2}{l}{ Ranking } \\
\cline { 2 - 4 } & $\xi_{1}$ & $\xi_{2}$ & $\xi_{3}$ & $\xi_{4}$ & \\
\hline IFWA $^{5,9}$ & Inaccessible & & & & $\times$ \\
PFWA $^{15}$ & 0.75078 & 0.71123 & 0.60872 & 0.66425 & $d_{1} \geq d_{2} \geq d_{4} \geq d_{3}$ \\
PFWA $^{20}$ & 0.66052 & 0.55713 & 0.40420 & 0.47255 & $d_{1} \geq d_{2} \geq d_{4} \geq d_{3}$ \\
SPFWA $^{20}$ & 0.53681 & 0.46357 & 0.28674 & 0.3795 & $d_{1} \geq d_{2} \geq d_{4} \geq d_{3}$ \\
GGPFWA $^{21}$ & 0.46035 & 0.21881 & 0.018209 & 0.11228 & $d_{1} \geq d_{2} \geq d_{4} \geq d_{3}$ \\
q-ROFWA $^{24}$ & 0.59987 & 0.47911 & 0.40693 & 0.41913 & $d_{1} \geq d_{2} \geq d_{4} \geq d_{3}$ \\
GGq- $\mathcal{R} O \mathcal{F}$ FA $^{20}$ & 0.41473 & 0.20370 & 0.072038 & 0.13362 & $d_{1} \geq d_{2} \geq d_{4} \geq d_{3}$ \\
\hline
\end{tabular}

\begin{tabular}{llllll}
\hline Operators & Score values & & & \multicolumn{2}{l}{ Ranking } \\
\cline { 2 - 4 } & $\xi_{1}$ & $\xi_{2}$ & $x i_{3}$ & $\xi_{4}$ & \\
\hline IFWA $^{5,9}$ & Inaccessible & & & $\times$ \\
PFWA $^{15,20}$ & Inaccessible & & & times \\
SPFWA $^{20}$ & Inaccessible & & & $\times$ \\
GGPFWA $^{21}$ & 0.46035 & 0.21881 & 0.018209 & 0.11228 & $d_{1} \geq d_{2} \geq d_{4} \geq d_{3}$ \\
q-ROFWA $^{24}$ & Inaccessible & & & & $\times$ \\
GGq- $\mathcal{R} O \mathcal{F}$ FA $^{\text {G }}$ & 0.41473 & 0.20370 & 0.072038 & 0.13362 & $d_{1} \geq d_{2} \geq d_{4} \geq d_{3}$
\end{tabular}

operator presented by Liu and Wang [21]. The ranking results of these aggregation operators are given in Table 4.

From the analysis the Table 4 it is clear that only IFWA operator by $\mathrm{Xu}$ [28] and $\mathrm{Li}$ [18] is inaccessible to rank the MCDM problem because it cannot tackle the assessment value satisfy $\mu_{d}+\eta_{d}>1$. The ranking result for the rest of MCDM methods remain same and the best optimal value is $d_{1}$. But the methods proposed by Yager [32], Ma and Xu [22] and Joshi [17] have also some restriction and they cannot handle the assessment value satisfy $\left(\mu_{d}\right)^{2}+\left(\eta_{d}\right)^{2}>1$. For example, if we make a minor change in Table 4 that is, by replace the alternatives $d_{11}, d_{24}, d_{32}$ and $d_{44}$ by $(0.9,0.85)$. Then the methods presented by by Yager [32], Ma and Xu [22] and Joshi [17] are also fail. However, the method presented in Liu and Wang [21] and the method developed in this paper still deal the situations by adjusting the value of $q$.

If we consider $\mathrm{GGq}-\mathcal{R O} \mathcal{F}$ expert's priority/preferences matrix $[\mathcal{Z}]_{m \times(n+k)}$ as given in Table 3, and utilize the methods proposed by by Yager [32], Ma and Xu [22] , Joshi [17] and Liu and Wang [21] on matrix $[\mathcal{Z}]_{m \times(n+k)}$, as their ranking result is shown in Table 5.
From Table 5, it is observed that the methods proposed by Yager [32], Ma and Xu [22] and Liu and Wang [21] are inaccessible to provide the ranking results and the method presented by Joshi [17] and our developed method is still working and produces the same result.

However, the method presented by Joshi [17] has some limitations and it cannot handle the assessment value satisfactorily $\left(\mu_{d}\right)^{2}+\left(\eta_{d}\right)^{2}>1$. For example, if we make a minor change in Table 5 that is, by replace just a single alternatives by $(0.9,0.8)$. Then, the method presented by Joshi [17] fails to handle the situation. However, the method developed in this paper is still works by adjusting the value of $q$. Thus IFWA operator by Xu [28] and Li [18], PFWA operator by Yager [32], Ma and Xu [22], GGPFWA operator by Joshi [17], and q-ROFWA operator Liu and Wang [21] are the special cases of the developed aggregation operators as shown in Remark 1, and Proposition 1. Finally from the above analysis and comparison, this fact is observed that the method proposed in this paper is more effective, powerful and superior to solve the MCDM problems than the existing methods. 


\section{Conclusion}

It has been observed, that in real-life situation provided information of a single expert are completely based on his own priority and may not lead to the accurate decisions. Therefore, acknowledging the initial preferences, it is necessary to justify the initially described preferences from other senior experts/judges to ensure the expert's level of trust and improve the accuracy of the final decision. This is only possible by adding the idea of generalized parameter to the original information. In this paper, the concept Gq-ROFSs is introduced by incorporating generalized parameter to the original information to the views of other senior decision makers or to the expertise of other senior decision makers in q-ROF environment. Then this idea explored to the group generalized parameter in which the preferences of two or more other senior experts/decision makers are analyzed in q-ROF environments. Different aggregation operators are presented on the basis of generalized parameter. Then, the defined aggregation operators are extended to GGq-ROF aggregation operators. These developed aggregations operators have the ability to adjust the situations in a better sequence on the basis of parameterization character. The major advantages of the developed concept is to reduce the probability of complexities, uncertainties and errors in the original information. The main focus of the developed work is on MCDM application by using the proposed approach. Finally, through comparative remark, it has been shown that the developed method is superior to the existing methods.

Acknowledgements The authors would like to thank the editor in chief, associate editor and the anonymous referees for detailed and valuable comments which helped to improve this manuscript.

\section{Compliance with ethical standards}

Conflict of interest The authors declare that they have no conflicts of interest regarding the publication of this paper.

Open Access This article is licensed under a Creative Commons Attribution 4.0 International License, which permits use, sharing, adaptation, distribution and reproduction in any medium or format, as long as you give appropriate credit to the original author(s) and the source, provide a link to the Creative Commons licence, and indicate if changes were made. The images or other third party material in this article are included in the article's Creative Commons licence, unless indicated otherwise in a credit line to the material. If material is not included in the article's Creative Commons licence and your intended use is not permitted by statutory regulation or exceeds the permitted use, you will need to obtain permission directly from the copyright holder. To view a copy of this licence, visit http://creativecomm ons.org/licenses/by/4.0/.

\section{References}

1. Ali MI (2018) Another view on q-rung orthopair fuzzy sets. Int J Intell Syst 33:2139-2153

2. Ali MI, Feng F, Mahmood T, Mahmood I, Faizan H (2019) A graphical method for ranking Atanassov's intuitionistic fuzzy values using the uncertainty index and entropy. Int $\mathbf{J}$ Intell Syst 34(10):2692-2712

3. Atanassov KT (1986) Intuitionistic fuzzy sets. Fuzzy Set Syst 20:87-96

4. Ding H, Hu X, Tang X (2019) Multiple-attribute group decision making for interval-valued intuitionistic fuzzy sets based on expert reliability and the evidential reasoning rule. Neural Comput Appl. https://doi.org/10.1007/s00521-019-04016-z

5. Feng F, Fujita H, Ali MI, Yager RR, Liu X (2018) Another View on generalized intuitionistic fuzzy soft sets and related multiattribute decision making methods. IEEE Trans Fuzzy Syst 27(3):474-488

6. Feng F, Liang M, Fujita H, Yager RR, Liu X (2019) Lexicographic orders of intuitionistic fuzzy values and their relationships. Mathematics 7(2): 166

7. Garg H, Arora R (2018) Generalized and group-based generalized intuitionistic fuzzy soft sets with applications in decision-making. Appl Intell 48:343-356

8. Garg H (2016) A new generalized Pythagorean fuzzy information aggregation using Einstein operations and its application to decision making. Int J Intell Syst 31:886-920

9. Garg H (2017) Generalized Pythagorean fuzzy geometric aggregation operators using Einstein t-norm and t-conorm for multi-criteria decision making process. Int J Intell Syst 32:597-630

10. Garg H, Kumar K (2019) A novel possibility measure to intervalvalued intuitionistic fuzzy set using connection number of set pair analysis and its applications. Neural Comput Appl. https://doi.org/ 10.1007/s00521-019-04291-w

11. Hayat K, Ali MI, Cao BY, Karaaslan F, Yang XP (2018) Another view of aggregation operators on group-based generalized intuitionistic fuzzy soft sets: multi-attribute decision making methods. Symmetry 10:753

12. Hussain A, Ali MI, Mahmood T (2019) Covering based q-rung orthopair fuzzy rough set model hybrid with TOPSIS for multiattribute decision making. J Intell Fuzzy Syst 37:981-993

13. Hussain A, Ali MI, Mahmood T (2020) Hesitant q-rung orthopair fuzzy aggregation operators with their applications in multi-criteria decision making. Iran J Fuzzy Syst 17(3):117-134

14. Hussain A, Ali MI, Mahmood T (2020) Pythagorean fuzzy soft rough sets and their applications in decision-making. J Taibah Univ Sci 14(1):101-113

15. Hussain A, Ali MI, Mahmood T, Munir M (2020) q-Rung orthopair fuzzy soft average aggregation operators and their application in multicriteria decision-making. Int J Intell Syst 35(4):571-599

16. Hussain A, Mahmood T, Ali MI (2019) Rough Pythagorean fuzzy ideals in semigroups. Comput Appl Math 38(2):67

17. Joshi BP (2019) Pythagorean fuzzy average aggregation operators based on generalized and group-generalized parameter with application in MCDM problems. Int J Intell Syst 34:895-919

18. Li DF (2014) Decision and game theory in management with intuitionistic fuzzy sets. Springer, Berlin, p 308

19. Liu P, Liu J (2018) Some q-rung orthopai fuzzy bonferroni mean operators and their application to multi-attribute group decision making. Int J Intell Syst 33:315-347

20. Liu P, Qin X (2019) A new decision-making method based on interval-valued linguistic intuitionistic fuzzy information. Cogn Comput 11(1):125-144

21. Liu P, Wang P (2018) Some q-rung orthopair fuzzy aggregation operators and their applications to multiple-attribute decision making. Int J Intell Syst 33:259-280

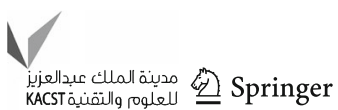


22. Ma ZM, Xu ZS (2016) Symmetric Pythagorean fuzzy weighted geometric/averaging operators and their application in multicriteria decision making problems. Int J Intell Syst 31:1198-1219

23. Mishra AR, Singh RK, Motwani D (2018) Intuitionistic fuzzy divergence measure-based ELECTRE method for performance of cellular mobile telephone service providers. Neural Comput Appl. https://doi.org/10.1007/s00521-018-3716-6

24. Peng X, Yang Y (2015) Some results for Pythagorean fuzzy sets. Int J Intell Syst 30:1133-1160

25. Peng X, Yuan H (2016) Fundamental properties of Pythagorean fuzzy aggregation operators. Fundam Inform 147:415-446

26. Xing Y, Zhang R, Zhou Z, Wang J (2019) Some q-rung orthopair fuzzy point weighted aggregation operators for multi-attribute decision making. Soft Comput 23(22):11627-11649

27. Xing Y, Zhang R, Wang J, Bai K, Xue J (2019) A new multi-criteria group decision-making approach based on q-rung orthopair fuzzy interaction Hamy mean operators. Neural Comput Appl. https:// doi.org/10.1007/s00521-019-04269-8

28. Xu ZS (2007) Intuitionistic fuzzy aggregation operators. IEEE Trans Fuzzy Syst 15:1179-1187

29. Xu Z, Zhao N (2016) Information fusion for intuitionistic fuzzy decision making: an overview. Inf Fusion 28:10-23

30. Yager RR (1988) On ordered weighted avergaing aggregation operators in multi-criteria decision making. IEEE Trans Syst Man Cybern 18:183-190

31. Yager RR (2013) Pythagorean fuzzy subsets. In: Proceedings of the Joint IFSA World Congress and NAFIPS Annual Meeting, Edmonton Canada. IEEE, pp 57-61

32. Yager RR (2014) Pythagorean membership grades in multicriteria decision making. IEEE Trans Fuzzy Syst 22:958-965
33. Yager RR (2016) Generalized orthopair fuzzy sets. IEEE Trans Fuzzy Syst 25(5):1222-1230

34. Yager RR, Abbasov AM (2013) Pythagorean membership grades, complex numbers and decision making. Int J Intell Syst 28:436452

35. Yager RR, Kacprzyk J (1997) The ordered weighted average operators: theory and applications. Kluwer Academic Publisher, Boston

36. Yu D (2014) Intuitionistic fuzzy information aggregation under confidence levels. Appl Soft Comput 19:147-160

37. Yu D (2015) A scientometrics review on aggregation operator research. Scientometrics 105:115-133

38. Zadeh LA (1965) Fuzzy sets. Inf Control 8:338-356

39. Zhang Y, Hu S, Zhou W (2019) Multiple attribute group decision making using J-divergence and evidential reasoning theory under intuitionistic fuzzy environment. Neural Comput Appl. https://doi. org/10.1007/s00521-019-04140-w

Publisher's Note Springer Nature remains neutral with regard to jurisdictional claims in published maps and institutional affiliations. 